\title{
Effects of Parameterized Diffusion on Simulated Hurricanes
}

\author{
RICHARD ROTUNNO AND GEORGE H. BRYAN \\ National Center for Atmospheric Research, * Boulder, Colorado
}

(Manuscript received 28 July 2011, in final form 9 March 2012)

\begin{abstract}
In this study the authors analyze and interpret the effects of parameterized diffusion on the nearly steady axisymmetric numerical simulations of hurricanes presented in a recent study. In that study it was concluded that horizontal diffusion was the most important control factor for the maximum simulated hurricane intensity. Through budget analysis it is shown here that horizontal diffusion is a major contributor to the angular momentum budget in the boundary layer of the numerically simulated storms. Moreover, a new scale analysis recognizing the anisotropic nature of the parameterized model diffusion shows why the horizontal diffusion plays such a dominant role. A simple analytical model is developed that captures the essence of the effect. The role of vertical diffusion in the boundary layer in the aforementioned numerical simulations is more closely examined here. It is shown that the boundary layer in these simulations is consistent with known analytical solutions in that boundary layer depth increases and the amount of "overshoot" (maximum wind in excess of the gradient wind) decreases with increasing vertical diffusion. However, the maximum wind itself depends mainly on horizontal diffusion and is relatively insensitive to vertical diffusion; the overshoot variation with vertical viscosity mainly comes from changes in the gradient wind with vertical viscosity. The present considerations of parameterized diffusion allow a new contribution to the dialog in the literature on the meaning and interpretation of the Emanuel potential intensity theory.
\end{abstract}

\section{Introduction}

Given the near axisymmetry of most mature hurricanes, the axisymmetric model continues to be the basis of analytical and numerical studies of hurricane intensity as a function of its environment (Emanuel 2004). While analytical models can give a clear picture of the interdependency among the variables characterizing a steadystate hurricane (e.g., Emanuel 1986, hereafter E86), quantitative estimates of maximum hurricane intensity for a given environment depend sensitively on the approximations needed to obtain analytical solutions (Persing and Montgomery 2003; Bryan and Rotunno 2009a, hereafter BR09a). On the other hand, numerical models, which make fewer approximations, do give definite predictions of maximum hurricane intensity as a function of environmental (external) parameters; however,

\footnotetext{
* The National Center for Atmospheric Research is sponsored by the National Science Foundation.

Corresponding author address: Richard Rotunno, National Center for Atmospheric Research, P.O. Box 3000, Boulder, CO 80307.

E-mail: rotunno@ucar.edu
}

those predictions depend on poorly known internal parameters representing principally the effects of turbulence and cloud microphysics. Bryan and Rotunno (2009b, hereafter BR09b), using an axisymmetric numerical model with fixed external parameters, conducted a systematic study of the dependence of simulated nearly steady hurricane intensity on the internal modeling parameters. BR09b found the strongest sensitivity of simulated structure and maximum intensity coming from the parameterized diffusion. In the present article, the authors take a closer look at this sensitivity through budget analysis of the BR09b numerical simulations and comparison of these with simpler fluid dynamical analogs.

By far the most studied effect of turbulent diffusion on hurricanes is that occurring in the hurricane boundary layer. The latter shares many features with the general class of boundary layer that forms at frictional boundaries normal to the rotation axis of a vortex flow $\left[0, V_{\infty}(r), 0\right]$ where the respective radial, azimuthal, and axial (vertical) velocity components $(u, v, w)$ refer to the system of cylindrical coordinates $(r, \phi, z)$. A general review of the problem may be found in Rott and Lewellen (1966); hence only the most important features are recalled here. In the steady-state vortex flow $\left[0, V_{\infty}(r), 0\right]$, the centrifugal force per unit mass $r^{-1} V_{\infty}^{2}(r)$ is balanced by 
the radial pressure gradient force per unit mass $-\rho^{-1} d p / d r$, where $\rho$ and $p$ represent the fluid density and pressure, respectively. However, near the rotation-axis-normal frictional boundary, the azimuthal velocity is reduced while the (inward-directed) radial pressure gradient force remains almost unchanged; the latter force must then be balanced by radial friction and acceleration, which in turn require radial inflow. This boundary layer radial inflow can be convergent, implying upward vertical motions that in turn may modify the assumed "interior" flow $\left[0, V_{\infty}(r), 0\right]$. In principle, a recalculation of the boundary layer with the modified interior flow is then required until both interior and boundary layer flows are mutually adjusted.

Numerical models such as the one examined in BR09b of course produce both the interior flow and boundary layer as components of one unified solution. In the BR09b numerical solutions, the effects of vertical diffusion in the boundary layer and interior are regulated by a specified vertical mixing length $l_{v}$; herein we examine the variation of the numerical solution features as a function of $l_{v}$ and show they are to a large degree consistent with classical rotating-flow boundary layer theory in which there is radial inflow, "overshooting" $\left[v(r, z)\right.$ in the boundary layer locally greater than $\left.V_{\infty}(r)\right]$, and upward flow from the boundary layer to the interior where the direct influence of boundary friction is small.

A major finding of BR09b is that the maximum simulated hurricane intensity is a weak function of $l_{v}$ but a strong function of the specified horizontal mixing length $l_{h}$. Consistently, the present analysis shows that the parameterized horizontal diffusion is a significant contributor to the budget of angular momentum in the hurricane boundary layer near the eyewall. Such horizontal diffusion effects have in the past not been considered significant since conventional boundary layer scale analysis, based on an isotropic eddy viscosity, holds that they are small (Batchelor 1967, section 5.7; Vogl and Smith 2009). However, to our knowledge all NWP models used in the study of hurricanes distinguish a horizontal and vertical eddy diffusivity (e.g., Skamarock et al. 2005, ch. 4) to ensure that horizontal diffusion is operative to prevent the formation of frontal discontinuities. Emanuel (1997) has shown that the hurricane eyewall is a type of front and, consistent with the latter, we find that horizontal diffusion is most important in the simulated eyewall in the boundary layer. ${ }^{1}$ We show here that this horizontal diffusion in the boundary layer is felt throughout the vortex as air parcels near the eyewall

\footnotetext{
${ }^{1}$ Observations (Marks et al. 2008) and large-eddy simulations of idealized hurricanes (Rotunno et al. 2009) support the idea that the eyewall in the hurricane boundary layer is particularly turbulent.
}

flowing upward out of the boundary layer adjust the radial distribution of $V_{\infty}(r)$ accordingly.

One feature of the hurricane boundary layer not shared with the prototypical rotating-flow boundary layer is the baroclinicity of the interior flow [i.e., $V_{\infty}=$ $\left.V_{\infty}(r, z)\right]$. E86 devised a model for $V_{\infty}(r, z)$ taking into account thermal wind balance, moist thermodynamics, and certain assumptions on the gross effects of vertical heat and angular momentum transport in the boundary layer. The present analysis allows a more precise interpretation of the role and nature of the simulated hurricane boundary layer in the E86 theory.

The present paper is organized as follows. Section 2 presents a scale analysis for numerical models applied to the hurricane. Section 3 examines some typical numerical solutions from BR09b as a function of the mixing lengths $\left(l_{h}, l_{v}\right)$ and presents analyses of the momentum budgets. Section 4 examines the solution response to variations of $l_{v}$ through the lens of classical rotating-flow boundary layer theory. Section 5 considers the BR09b numerical solution response to variations of $l_{h}$ and develops a simple analytical model that qualitatively captures the important effect of horizontal diffusion on the maximum simulated azimuthal wind speed. Section 6 reconsiders the E86 theory (and its extension in BR09a) in light of the present considerations of the effects of parameterized diffusion. Section 7 provides a comparison of the simulated boundary layers in the BR09b simulations with some recent observations. Section 8 provides a summary of the main points.

\section{Scale analysis of the governing equations}

\section{a. Nondimensionalized form of the simplified set}

The full governing equations are given in section 2 of BR09b; here we consider only the equations for $u, w$, angular momentum $m(=r v)$, buoyancy $b$, and continuity in their Boussinesq, anelastic form in order to isolate cleanly the effects of parameterized diffusion. Furthermore, we will focus on the inner region of the hurricane where velocities are large enough, and horizontal length scales small enough, to neglect the Coriolis accelerations. With horizontal velocities nondimensionalized by $V$ (a characteristic interior velocity), radius by $\lambda_{h}$ (the distance over which the interior velocity field undergoes significant variation), height by the vertical scale $\lambda_{v}$, vertical velocity by $\left(\lambda_{v} / \lambda_{h}\right) V$, the pressure variable $\phi$ by $V^{2}$, and buoyancy by $V^{2} / \lambda_{v}$, the nondimensional governing equations are

$$
\frac{d u}{d t}=-\frac{\partial \phi}{\partial r}+\frac{m^{2}}{r^{3}}+D_{u}
$$




$$
\begin{gathered}
\alpha^{2} \frac{d w}{d t}=-\frac{\partial \phi}{\partial z}+b+D_{w}, \\
\frac{d m}{d t}=D_{m}, \\
\frac{d b}{d t}=D_{b}, \\
\frac{1}{r} \frac{\partial r u}{\partial r}+\frac{\partial w}{\partial z}=0
\end{gathered}
$$

where $\alpha \equiv \lambda_{v} / \lambda_{h}$ and

$$
\begin{aligned}
D_{u}= & \frac{1}{\operatorname{Re}_{h}}\left[\frac{1}{r} \frac{\partial}{\partial r}\left(2 f_{h} r \frac{\partial u}{\partial r}\right)-2 f_{h} \frac{u}{r^{2}}\right] \\
& +\frac{1}{\alpha^{2} \operatorname{Re}_{v}} \frac{\partial}{\partial z}\left[f_{v}\left(\frac{\partial u}{\partial z}+\alpha^{2} \frac{\partial w}{\partial r}\right)\right] \\
D_{w}= & \frac{1}{\operatorname{Re}_{v}} \frac{1}{r} \frac{\partial}{\partial r}\left[f_{v} r\left(\frac{\partial u}{\partial z}+\alpha^{2} \frac{\partial w}{\partial r}\right)\right]+\frac{1}{\operatorname{Re}_{v}} \frac{\partial}{\partial z}\left(2 f_{v} \frac{\partial w}{\partial z}\right) \\
D_{m}= & \frac{1}{\operatorname{Re}_{h}} \frac{1}{r} \frac{\partial}{\partial r}\left(f_{h} r^{3} \frac{\partial}{\partial r} \frac{m}{r^{2}}\right)+\frac{1}{\alpha^{2} \operatorname{Re}_{v}} \frac{\partial}{\partial z}\left(f_{v} \frac{\partial m}{\partial z}\right) \\
D_{b}= & \frac{1}{\operatorname{Re}_{h}} \frac{1}{r} \frac{\partial}{\partial r}\left(f_{h} r \frac{\partial b}{\partial r}\right)+\frac{1}{\alpha^{2} \operatorname{Re}_{v}} \frac{\partial}{\partial z}\left(f_{v} \frac{\partial b}{\partial z}\right)
\end{aligned}
$$

The horizontal and vertical Reynolds numbers are defined as

$$
\operatorname{Re}_{h}=V \lambda_{h} / \tilde{\nu}_{h}, \quad \operatorname{Re}_{v}=V \lambda_{h} / \tilde{\nu}_{v}
$$

where $\left(\tilde{\nu}_{h}, \tilde{\nu}_{v}\right)$ denote the maximum eddy viscosities, and all spatial variation in the eddy viscosities is absorbed in the $O(1)$ nondimensional functions $f_{h}$ and $f_{v}$; that is, $\left(\nu_{h}, \nu_{v}\right)=\left(\tilde{\nu}_{h} f_{h}, \tilde{\nu}_{v} f_{v}\right)$. The eddy viscosities $\left(\nu_{h}, \nu_{v}\right)$ are parameterized by standard mixing length theory and are given by BR09b's (16) and (17); from these it may be deduced that

$$
\begin{aligned}
& \tilde{\nu}_{h}=l_{h}^{2} \tilde{S}_{h}, \quad \tilde{\nu}_{v}=l_{v}^{2} \tilde{S}_{v}, \\
& f_{h}=S_{h} / \tilde{S}_{h}, \quad f_{v}=\left(S_{v} / \tilde{S}_{v}\right) \sqrt{1-\mathrm{Ri},}
\end{aligned}
$$

where $\tilde{S}_{h}$ and $\tilde{S}_{v}$ are the maximum values of the horizontal and vertical deformations, respectively, and $\mathrm{Ri}$ is the Richardson number.

\section{b. Scale analysis}

In classical boundary layer analysis (e.g., Batchelor 1967, p. 306), $\operatorname{Re}_{h}=\mathrm{Re}_{v}=\mathrm{Re} \equiv V \lambda_{h} / \nu$ and $m=b=0$. Accommodation of the frictional lower boundary conditions requires the retention of the term involving vertical derivatives in (6); therefore one sets $\alpha=1 / \sqrt{\mathrm{Re}}$. In the limit $\operatorname{Re} \rightarrow \infty$, the boundary layer equations consist of (1) with $D_{u}=\partial_{z}\left(f_{v} \partial_{z} u\right)$, (5), and (2) with (7) (indicating $\partial_{z} \phi=0$ ).

In weather and climate models (where $m, b \neq 0$ ) one often encounters sharp horizontal gradients (e.g., fronts); therefore, all (to our knowledge) such models treat horizontal diffusion differently than they do vertical diffusion. To prevent gradients in simulated fields from falling below the grid resolution, the horizontal viscosity is chosen so that in effect $\operatorname{Re}_{h} \sim O(1)$ and the variable function $f_{h}$ in (11) ensures that horizontal diffusion is active only in places where the horizontal velocity gradients are large. To account for frictional boundary conditions at the lower surface, one lets $\alpha=1 / \sqrt{\operatorname{Re}_{v}}$; in the limit $\operatorname{Re}_{v} \rightarrow \infty$, (2) now reduces to hydrostatic balance and, in addition to the terms involving only vertical derivatives, there now remain the first terms on the right-hand sides of (6), (8), and (9), which represent horizontal diffusion.

The requirement that $\mathrm{Re}_{h} \sim O(1)$ readily gives an estimate for $\lambda_{h}$ : substituting the first term of (11) into the first term of (10) and estimating $\tilde{S}_{h} \sim V / \lambda_{h}$ yields

$$
\lambda_{h} \sim l_{h}
$$

\section{Solution features and budget analysis}

\section{a. Solution dependence on $\left(l_{h}, l_{v}\right)$}

To carry out a detailed analysis of vertical structure, in the present paper we have run the suite of simulations in BR09b at much higher vertical resolution (the vertical grid interval varies from $20 \mathrm{~m}$ at $z=0 \mathrm{~km}$ to $250 \mathrm{~m}$ at $z=6.5 \mathrm{~km}$ ) and show the results in Figs. 1a-c, which, in addition to the nearly steady-state (8-10-day averaged) maximum (dimensional) azimuthal velocity, include its radius $\hat{r}_{m}$ and height $\hat{z}_{m} .{ }^{2}$ Comparison of Fig. 1a with the results of BR09b indicates little quantitative change with higher vertical resolution and the same qualitative conclusion that $\hat{v}_{m}$ has little variation with $l_{v}$ but is a strong function of $l_{h}$. Herein we examine the flow structure underlying the data.

Figure 2 shows a representation of the radial-vertical velocity vectors and the angular momentum field for the innermost $100 \mathrm{~km}$ and lowermost $8 \mathrm{~km}$ for four selected simulations. For $l_{v}=200 \mathrm{~m}$, comparison of the $l_{h}=$ $1500 \mathrm{~m}$ case (Fig. 2a) with the $l_{h}=3000 \mathrm{~m}$ case (Figs. $2 b)$ shows the strong effect horizontal diffusion has on

\footnotetext{
${ }^{2}$ Dimensional independent and dependent variables are henceforth denoted by the hat symbol.
} 


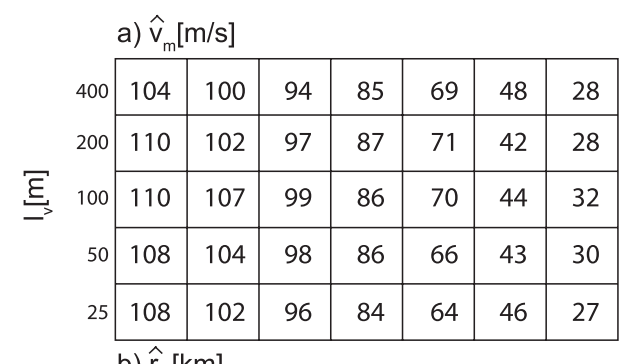

\begin{tabular}{|c|c|c|c|c|c|c|c|}
\hline \multirow[b]{2}{*}{400} & \multicolumn{7}{|c|}{ b) $\hat{r}_{m}[\mathrm{~km}]$} \\
\hline & 13 & 14 & 15 & 18 & 22 & 35 & 72 \\
\hline 200 & 13 & 14 & 15 & 17 & 21 & 40 & 69 \\
\hline 100 & 12 & 13 & 14 & 17 & 22 & 37 & 62 \\
\hline 50 & 11 & 12 & 13 & 16 & 21 & 37 & 61 \\
\hline 25 & 11 & 12 & 13 & 15 & 20 & 33 & 57 \\
\hline
\end{tabular}

c) $\hat{z}_{m}[\mathrm{~km}]$

\begin{tabular}{|c|c|c|c|c|c|c|c|}
\hline 400 & 1.1 & 1.1 & 1.1 & 1.3 & 1.3 & 1.3 & 1.5 \\
\hline 200 & 1.0 & 1.0 & 1.1 & 1.1 & 1.0 & 1.1 & 1.2 \\
\hline 100 & 0.8 & 0.9 & 0.9 & 1.0 & 0.9 & 0.9 & 1.0 \\
\hline 50 & 0.6 & 0.7 & 0.7 & 0.8 & 0.7 & 0.8 & 0.8 \\
\hline 25 & 0.4 & 0.5 & 0.5 & 0.5 & 0.5 & 0.5 & 0.5 \\
\hline & 94 & 188 & 375 & $\begin{array}{l}750 \\
{[\mathrm{~m}]}\end{array}$ & 1500 & 3000 & 6000 \\
\hline
\end{tabular}

d) $\hat{\mathrm{v}}_{\mathrm{g}, \mathrm{m}}[\mathrm{m} / \mathrm{s}]$

\begin{tabular}{|l|l|l|l|l|l|l|}
\hline 98 & 93 & 87 & 78 & 65 & 45 & 26 \\
\hline 97 & 90 & 83 & 76 & 65 & 39 & 26 \\
\hline 88 & 85 & 77 & 69 & 60 & 40 & 29 \\
\hline 77 & 73 & 71 & 60 & 53 & 37 & 26 \\
\hline 74 & 68 & 62 & 57 & 49 & 36 & 23 \\
\hline
\end{tabular}

e) $v_{m}^{B R}$

\begin{tabular}{|l|l|l|l|l|l|l|}
\hline 1.09 & 1.08 & 1.09 & 1.08 & 1.05 & 1.06 & 1.11 \\
\hline 1.13 & 1.13 & 1.17 & 1.14 & 1.09 & 1.09 & 1.10 \\
\hline 1.24 & 1.25 & 1.29 & 1.25 & 1.16 & 1.12 & 1.12 \\
\hline 1.40 & 1.42 & 1.39 & 1.43 & 1.24 & 1.18 & 1.15 \\
\hline 1.45 & 1.50 & 1.54 & 1.48 & 1.31 & 1.26 & 1.19 \\
\hline
\end{tabular}

f) $\mathrm{E}-\mathrm{PI}[\mathrm{m} / \mathrm{s}]$

\begin{tabular}{|l|l|l|l|l|l|l|}
\hline 76 & 75 & 73 & 67 & 59 & 52 & 52 \\
\hline 75 & 73 & 69 & 64 & 58 & 52 & 52 \\
\hline 71 & 69 & 66 & 62 & 59 & 52 & 49 \\
\hline 67 & 65 & 62 & 59 & 54 & 50 & 46 \\
\hline 63 & 61 & 59 & 55 & 50 & 48 & 42 \\
\hline 94 & 188 & 375 & 750 & 1500 & 3000 & 6000 \\
7 &
\end{tabular}

FIG. 1. Nearly steady-state (8-10-day average) data from the BR09b numerical model for (a) maximum azimuthal velocity $\hat{v}_{m}\left(\mathrm{~m} \mathrm{~s}^{-1}\right)$; (b) its radius $\hat{r}_{m}(\mathrm{~km}) ;(\mathrm{c})$ its height $\hat{z}_{m}(\mathrm{~km}) ;(\mathrm{d}) V_{g, m}$ $\left(\mathrm{m} \mathrm{s}^{-1}\right)$, the gradient wind at $\left(\hat{r}_{m}, \hat{z}_{m}\right)$; (e) $v_{m}^{\mathrm{BR}}$ (the superscript BR signifies the $v_{m}$ analyzed from the BR09b simulations), the maximum azimuthal wind nondimensionalized by $V_{g, m}$; and (f) E-PI $\left(\mathrm{m} \mathrm{s}^{-1}\right)$ (calculated as in BR09a) as functions of horizontal and vertical mixing lengths, $l_{h}(\mathrm{~m})$ and $l_{v}(\mathrm{~m})$, respectively.

the radial distribution of $\hat{m}$ and therefore on $\hat{v}_{m}$ and $\hat{r}_{m}$. In both cases, however, $\hat{z}_{m}$ is nearly identical; moreover, the vertical structure of the velocity field near $\hat{r}=\hat{r}_{m}$ is characterized by an oscillation whose wavelength is nearly the same in each case. With $l_{v}$ reduced to $50 \mathrm{~m}$, Figs. $2 \mathrm{c}$ and $2 \mathrm{~d}$ indicate that $\hat{z}_{m}$ is reduced as well as the vertical wavelength of the above described oscillation. As in the case with $l_{v}=200 \mathrm{~m}$, Figs. $2 \mathrm{c}$ and $2 \mathrm{~d}$ indicate a significantly smoother radial distribution of $\hat{m}$, a decrease in $\hat{v}_{m}$, and an increase in $\hat{r}_{m}$ with increasing $l_{h}$.

\section{b. Budget analysis}

In this section we examine the balances in the steadystate equations for $\hat{u}$ and $\hat{m}$ choosing the case $l_{h}=1500 \mathrm{~m}$ and $l_{v}=200 \mathrm{~m}$ as a representative example. Figure 3 is constructed so that the left column contains the total derivative of $\hat{u}$ (Fig. 3a) and its contributors (Figs. 3b-d) while the right column contains the total derivative of $\hat{m}$ (Fig. 3e) and its contributors (Figs. 3f,g); Fig. 3h shows three selected streamlines. Beginning with the budget for $d \hat{u} / d \hat{t}$, Fig. $3 \mathrm{~d}$ shows the sum of the first two terms on the rhs of (1). ${ }^{3}$ Figure $3 \mathrm{~d}$ indicates that beyond $\hat{r}=\hat{r}_{m}$, and in the lowermost kilometer, the radial pressure gradient accelerates the flow inward; Fig. 3 b shows that the latter inward acceleration is largely balanced by vertical diffusion. Near $\hat{r}=\hat{r}_{m}$, Fig. 3d indicates that the centrifugal acceleration overcomes the inward pressure gradient acceleration; at steady state, this outward acceleration largely accounts for the total acceleration $\left(\hat{u} \partial_{\hat{r}} \hat{u}+\hat{w} \partial_{\hat{z}} \hat{u}\right)$ (Fig. 3a) with a small contribution from horizontal diffusion (Fig. 3c). Note that while $\left(\hat{m}^{2} / \hat{r}^{3}-\partial_{\hat{r}} \hat{\phi}\right)_{\max } \approx 0.041 \mathrm{~m} \mathrm{~s}^{-2}$, the individual terms at that location, $\hat{m}^{2} / \hat{r}^{3} \approx 0.253 \mathrm{~m} \mathrm{~s}^{-2}$ and $\partial_{\hat{r}} \hat{\phi} \approx 0.212 \mathrm{~m} \mathrm{~s}^{-2}$, so that the flow is, to a first approximation, in cyclostrophic balance.

\footnotetext{
${ }^{3}$ The present analysis uses the exact equations as described in BR09a; we have verified that the Coriolis acceleration is negligible as assumed in (1).
} 

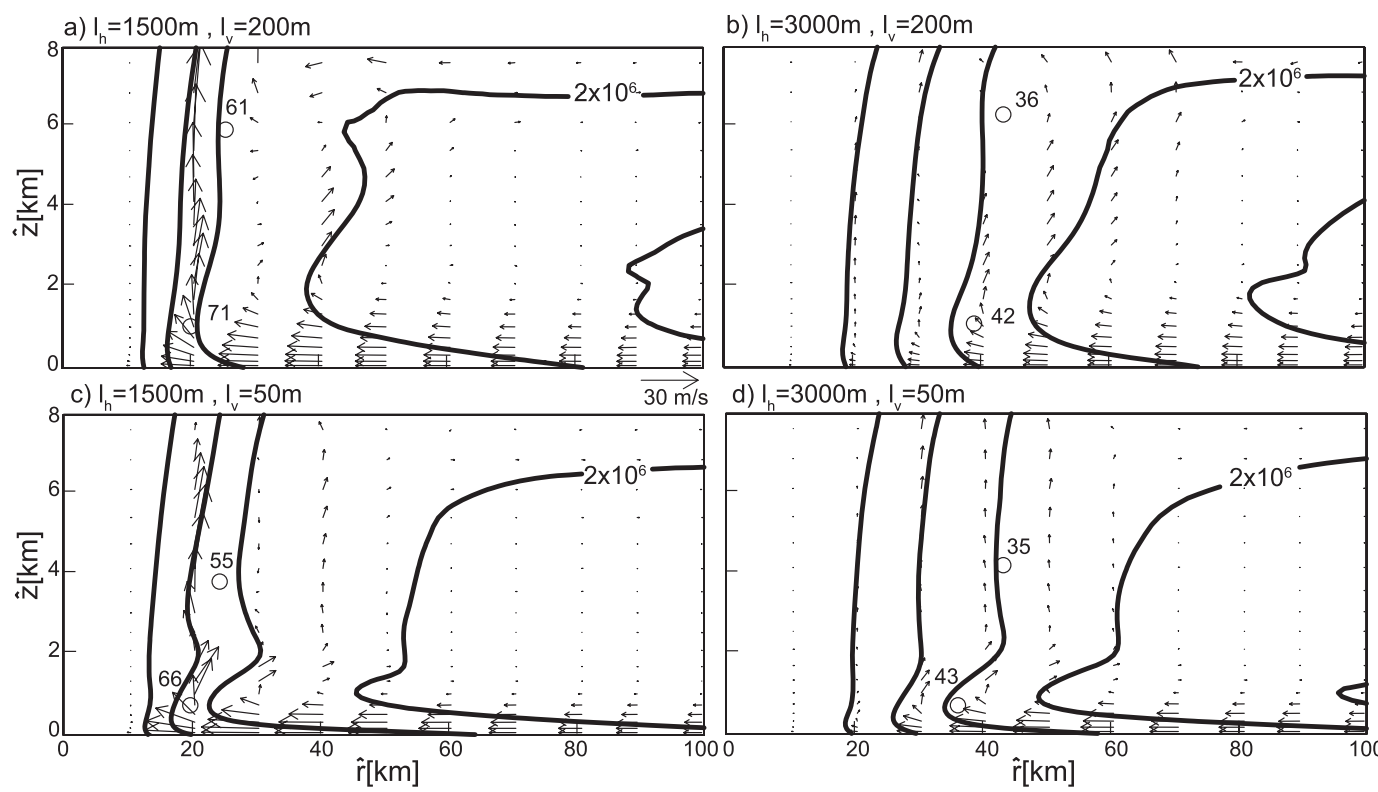

d) $I_{h}=3000 \mathrm{~m}, I_{v}=50 \mathrm{~m}$

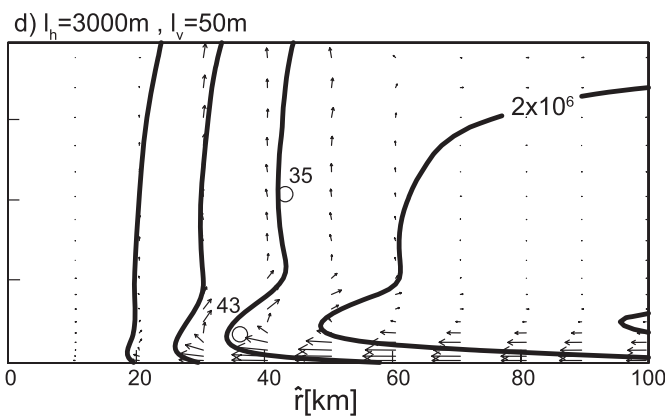

FIG. 2. Nearly steady-state angular momentum $\hat{m}$ [thick solid lines; contour interval $\left.(\mathrm{CI})=0.5 \times 10^{6} \mathrm{~m}^{2} \mathrm{~s}^{-1}\right)$ and radialvertical wind vectors $(\hat{u}, \hat{w})$ for $(\mathrm{a})\left(l_{h}, l_{v}\right)=(1500 \mathrm{~m}, 200 \mathrm{~m}),(\mathrm{b})\left(l_{h}, l_{v}\right)=(3000 \mathrm{~m}, 200 \mathrm{~m}),(\mathrm{c})\left(l_{h}, l_{v}\right)=(1500 \mathrm{~m}, 50 \mathrm{~m})$, and $(\mathrm{d})\left(l_{h}, l_{v}\right)=(3000 \mathrm{~m}, 50 \mathrm{~m})$. The open circles mark the local maxima of $\hat{v}$ with the values indicated in $\mathrm{m} \mathrm{s}^{-1}$.

Figure $3 \mathrm{e}$ shows that $d \hat{m} / d \hat{t}$ is largely negative, indicating the loss of angular momentum for fluid parcels traveling in the vicinity of $\hat{r}=\hat{r}_{m}$ (Fig. 3h); comparison of Figs. $3 \mathrm{f}$ and $3 \mathrm{~g}$ shows that horizontal diffusion is a major contributor. Beyond $\hat{r}=\hat{r}_{m}$, horizontal diffusion is small compared with vertical diffusion, which is confined to the lowermost kilometer or so. For further reference we show in Figs. $4 \mathrm{a}$ and $4 \mathrm{~b}$ the individual terms $\hat{u} \partial_{\hat{r}} \hat{m}$ and $\hat{w} \partial_{\hat{z}} \hat{m}$ contributing to $d \hat{m} / d \hat{t}$.

\section{Analysis of solution variation with $l_{v}$}

Although both horizontal and vertical diffusion are important contributors to the solutions under study, in this and the following section we look at simple fluid dynamical analogs treating each effect separately with the goal of isolating the features that uniquely attach to each. In this section we consider just the effects of vertical diffusion and hence the traditional boundary layer equations (section 2) apply.

All existing analytical solutions for the boundary layer of a rotating flow assume negligible buoyancy effects in the boundary layer, constant viscosity, and zero horizontal diffusion. In the typical analysis, there is a given interior flow $V_{\infty}(r)$ at a large distance from the lower (frictional) boundary. The qualitative behavior of the boundary layer solution was described in section 1 but only a few analytical solutions exist for $V_{\infty}(r)$ relevant to the hurricane. Perhaps the most ambitious analytical attack on the problem was made by (Kuo 1971, hereafter
K71); we defer to his excellent summary of the rotatingflow boundary layer problem and only highlight a few of the features we find most relevant to the current analysis. The object of the K71 analysis was the boundary layer beneath a vortex characterized by a strong (weak) increase of $m_{\infty}(r)$ [ $\left.=r V_{\infty}(r)\right]$ with $r$ in the inner (outer)core region. The similarity of the K71 vortex to the distributions of $\hat{m}(r)$ above the boundary layer found in the BR09b numerical simulations (Fig. 2) motivates the present revisiting of the K71 study.

\section{a. Inner-core region $\left(\hat{r} \approx \hat{r}_{m}\right)$}

Most relevant to the inner-core region are power-law vortices of the type $V_{\infty}(r)=r^{n}$. Here we focus for simplicity on the case $n=1$ (solid-body rotation), which was first solved by U. T. Bödewadt in 1940 and discussed in detail by (Schlichting 1968, ch. 11). ${ }^{4}$ The Bödewadt solution using the present nondimensionalization and notation is

$$
\begin{aligned}
(u, v, w) & =[r F(z), r G(z), H(z)], \\
z & =\hat{z} / \lambda_{v}, \quad \lambda_{v}=\sqrt{v_{v} / \omega},
\end{aligned}
$$

where $\omega$ is the constant (solid body) rotation rate. Setting $\omega=V / \lambda_{h}$, the last term of (13) is equivalent to the

\footnotetext{
${ }^{4}$ Similarity solutions to the Navier-Stokes equations of this kind belong to a general category known as von Kármán swirling flows (Zandbergen and Dijkstra 1987); their realizability has been confirmed by numerical simulations over finite-radius discs.
} 

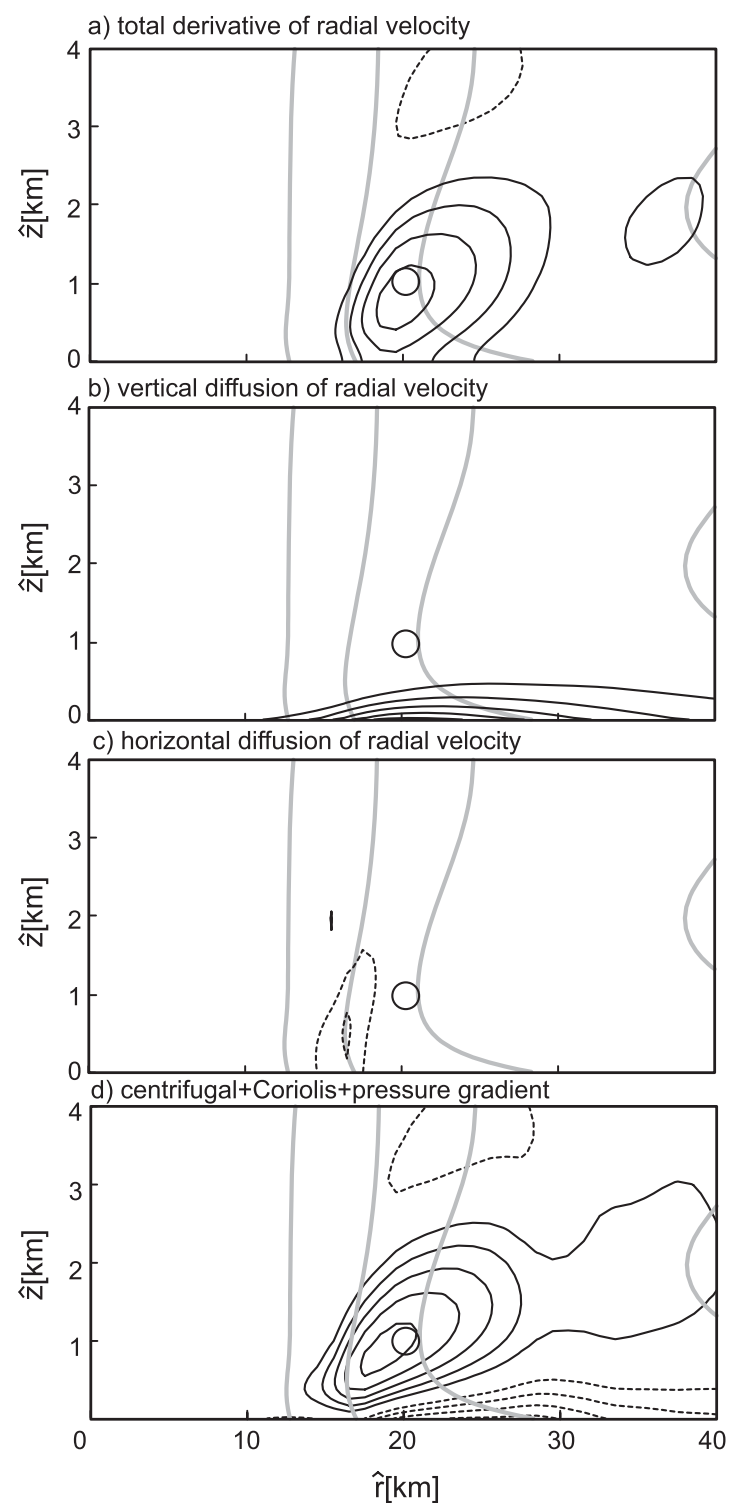

e) total derivative of angular momentum

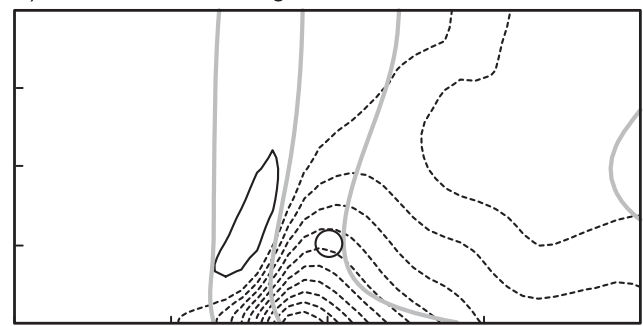

f) vertical diffusion of angular momentum

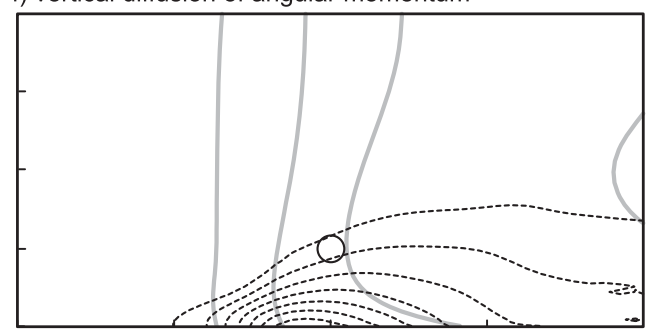

g) horizontal diffusion of angular momentum

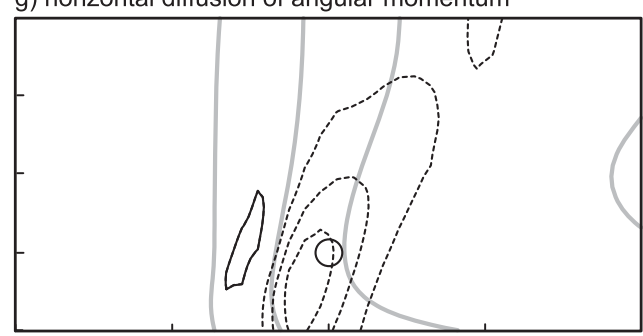

h) streamlines

FIG. 3. Budget analysis for the radial velocity $\hat{u}$ and angular momentum $\hat{m}$ for the case $\left(l_{h}, l_{v}\right)=(1500 \mathrm{~m}, 200 \mathrm{~m})$ showing (a) $d \hat{u} / d \hat{t}$; (b) the vertical diffusion of $\hat{u}$; (c) the horizontal diffusion of $\hat{u}$; (d) the sum of the centrifugal, Coriolis, and pressure gradient accelerations; (e) $d \hat{m} / d \hat{t}$; (f) the vertical diffusion of $\hat{m}$; and (g) the horizontal diffusion of $\hat{m}$. (h) Three selected streamlines. In (a)-(d), CI $=0.0075 \mathrm{~m} \mathrm{~s}^{-2}$ and in (e)-(g), CI $=75 \mathrm{~m}^{2} \mathrm{~s}^{-2}$ with negative values indicated by dashed lines (zero contour not plotted). The solid gray lines are contours of $\hat{m} ; \mathrm{CI}=$ $0.5 \times 10^{6} \mathrm{~m}^{2} \mathrm{~s}^{-1}$. The open circle marks the location of $\hat{v}_{m}$.

requirement $\alpha=1 / \sqrt{\operatorname{Re}_{v}}$ and it may be verified that the nondimensional variables $(u, v, w)$ and $(r, z)$ are the same as those used in section 2. Figure 5a shows the classic Bödewadt boundary layer profiles characterized by the low-level inflow, rising motion, and an azimuthal velocity that overshoots the cyclostrophically balanced solution to which it tends as $z \rightarrow \infty$. We observe through (13) that the viscosity only determines the dimensional vertical scale of the solution and has no effect on the maximum azimuthal velocity $r G_{\max }$. The budgets for $u$ and $m$ are shown in Figs. 5b and 5c and indicate a broad similarity with the budget analysis of the numerical solutions shown in Fig. 3 in the region near $\hat{r}=\hat{r}_{m}$.

A major difference between the Bödewadt boundary layer and those characteristic of hurricanes is the lower boundary condition. K71 argued that the partial-slip condition (Taylor 1915),

$$
(u, v)=K\left(\partial_{z} u, \partial_{z} v\right)
$$


a) horizontal advection of angular momentum

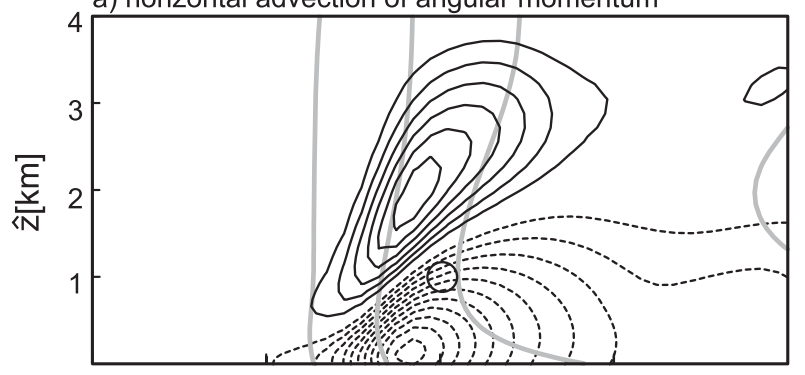

b) vertical advection of angular momentum

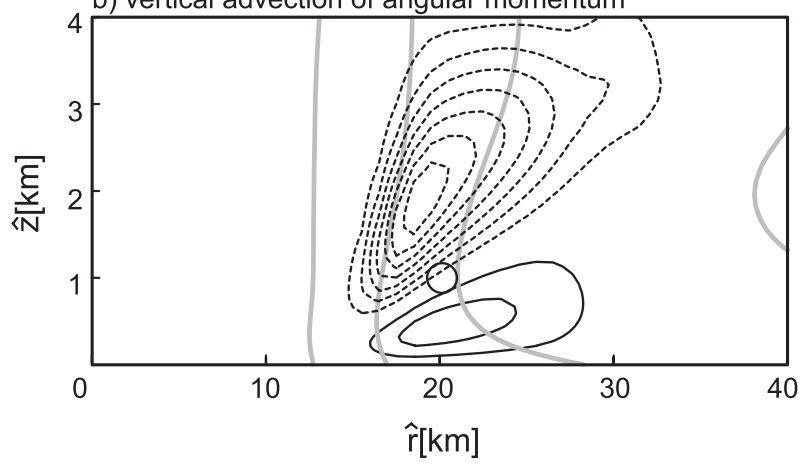

FIG. 4. The terms (a) $\hat{u} \partial_{r} \hat{m}$ and (b) $\hat{w} \partial_{z} \hat{m} ; \mathrm{CI}=75 \mathrm{~m}^{2} \mathrm{~s}^{-2}$, with negative values indicated by dashed lines (zero contour not plotted). The solid gray lines are contours of $\hat{m} ; \mathrm{CI}=0.5 \times 10^{6} \mathrm{~m}^{2} \mathrm{~s}^{-1}$. The open circle marks the location of $\hat{v}_{m}$.

applied at $z=0$ would produce solutions more in line with observations. This boundary condition has the attractive feature that the Bödewadt solution with a noslip condition at the surface is produced with $K=$ 0 whereas, in the limit $K \rightarrow \infty$, (14) becomes a free-slip condition with the solution $(F, G, H)=(0,1,0)$. We have reproduced and extended K71's results over the range $0 \leq K \leq 4$ (details are given in the appendix and results summarized in Table 1) and show the solution in Fig. 5d for the case $K=1$. Aside from the nonzero values of the horizontal velocity $(F, G)$ at the surface, the most notable features are the reductions in the inflow $\left(-F_{\min }\right)$, rising motion $\left(H_{\max }\right)$, and overshoot $\left(G_{\max }-1\right)$. Although the heights of these extrema decrease with increasing $K$, the wavelength of the vertical oscillation is relatively independent of $K{ }^{5}$ Figures $5 \mathrm{e}$ and $5 \mathrm{f}$ indicate that the budgets for $u$ and $m$ with $K=1$ are qualitatively similar to their $K=0$ counterparts.

The most important feature of the BR09b-solution boundary layer is the dependence of $\hat{v}_{m}$ and $\hat{z}_{m}$ on $\left(l_{h}, l_{v}\right)$

\footnotetext{
${ }^{5}$ This suggests that the dimensional vertical wavelength of the oscillation should vary in proportion to $\sqrt{\nu_{v} / \omega}$ for any $K$. Since we expect $\nu_{v}$ to increase with $l_{v}$ in the BR09b simulations, the increase with $l_{v}$ of the vertical scale of the oscillations seen in Fig. 2 is consistent with the K71 theory.
}

(Figs. 1a,c). To compare this behavior with that of the K71 solutions (Table 1), we need to relate the nondimensional parameter $K$ in (14) to the dimensional parameters used in the BR09b numerical simulations. The dimensional form of (14) implies $K=\hat{K} / \lambda_{v}=$ $\hat{K} / \sqrt{\nu_{v} / \omega}$, where $\hat{K}$ represents a second vertical scale. For a lower boundary condition that is compatible with (14), we approximate the surface stress used in BR09b, $C_{D} \sqrt{\hat{u}^{2}+\hat{v}^{2}}(\hat{u}, \hat{v})$, by $C_{D}\left(V_{0}\right) V_{0}(\hat{u}, \hat{v})$ and equate the latter to the internal surface stress $\nu_{v} \partial_{\hat{z}}(\hat{u}, \hat{v})$ so that

$$
\hat{K}=\nu_{v} / C_{D} V_{0}
$$

and therefore

$$
K=\frac{\sqrt{\nu_{v} \omega}}{C_{D} V_{0}}
$$

where $C_{D}\left(V_{0}\right)$ is the drag coefficient and $V_{0}$ is the surface wind speed. Our analysis (not shown) of the BR09b simulations indicates that for fixed $l_{h}$, the variables $\omega, V_{0}$, and $C_{D}$ vary only slightly with $l_{v}$ (consistent with Fig. 1 a); hence $K$ increases with $\nu_{v}$ and, by the second term of (11), with $l_{v}$. Moreover, for fixed $l_{v}$, our analysis indicates that $K$ is not very sensitive to variations in $l_{h}$ and that therefore the BR09b-solution dependence on $l_{h}$ is outside the reach of the K71 boundary layer theory. In short, the BR09b simulations indicate that $K$ is an increasing function of $l_{v}$ and has no significant variation with $l_{h}$.

Turning first to the dimensional height of the maximum azimuthal wind speed $\hat{z}_{m}=\sqrt{\nu_{v} / \omega} z_{m}(K)$, we use (16) to substitute for $\nu_{v}$ to obtain

$$
\hat{z}_{m}(K)=\left(C_{D} V_{0} / \omega\right) K z_{m}(K) .
$$

As mentioned above, the parameters $\omega, V_{0}$, and $C_{D}$ vary little for fixed $l_{h}$; hence, as seen in Table $1, K z_{m}(K)$ increases with increasing $K$ (and thus $l_{v}$ ) and therefore the $\mathrm{K} 71 \hat{z}_{m}$ is expected to increase with $l_{v}$, consistent with the BR09a numerical solutions (Fig. 1c).

The dimensional maximum azimuthal wind speed $\hat{v}_{m}=V v_{m}(K)$ is more difficult to determine since it depends on a "given" interior flow velocity scale $V$ as well as on the parameter $K$. If we take $V=V_{g, m}$, the gradient wind at $\left(\hat{r}_{m}, \hat{z}_{m}\right)$, then we need to know $V_{g, m}\left(l_{h}, l_{v}\right)$ in order to compare the K71 theory with the BR09b simulations; Figs. 1d-e show $V_{g, m}\left(l_{h}, l_{v}\right)$ and $v_{m}^{\mathrm{BR}}=\hat{v}_{m} / V_{g, m}\left(l_{h}, l_{v}\right)$. Comparing Fig. 1e for $l_{h}$ fixed with Table 1, we observe that $v_{m}^{\mathrm{BR}}$ decreases with increasing $l_{v}$ (increasing $K$ ) and therefore is consistent with the K71 theory. We have not yet found an explanation for the variation of $V_{g, m}$ with $l_{v}$ but suspect it occurs through boundary layer effects on the thermodynamics as well as the dynamics. 

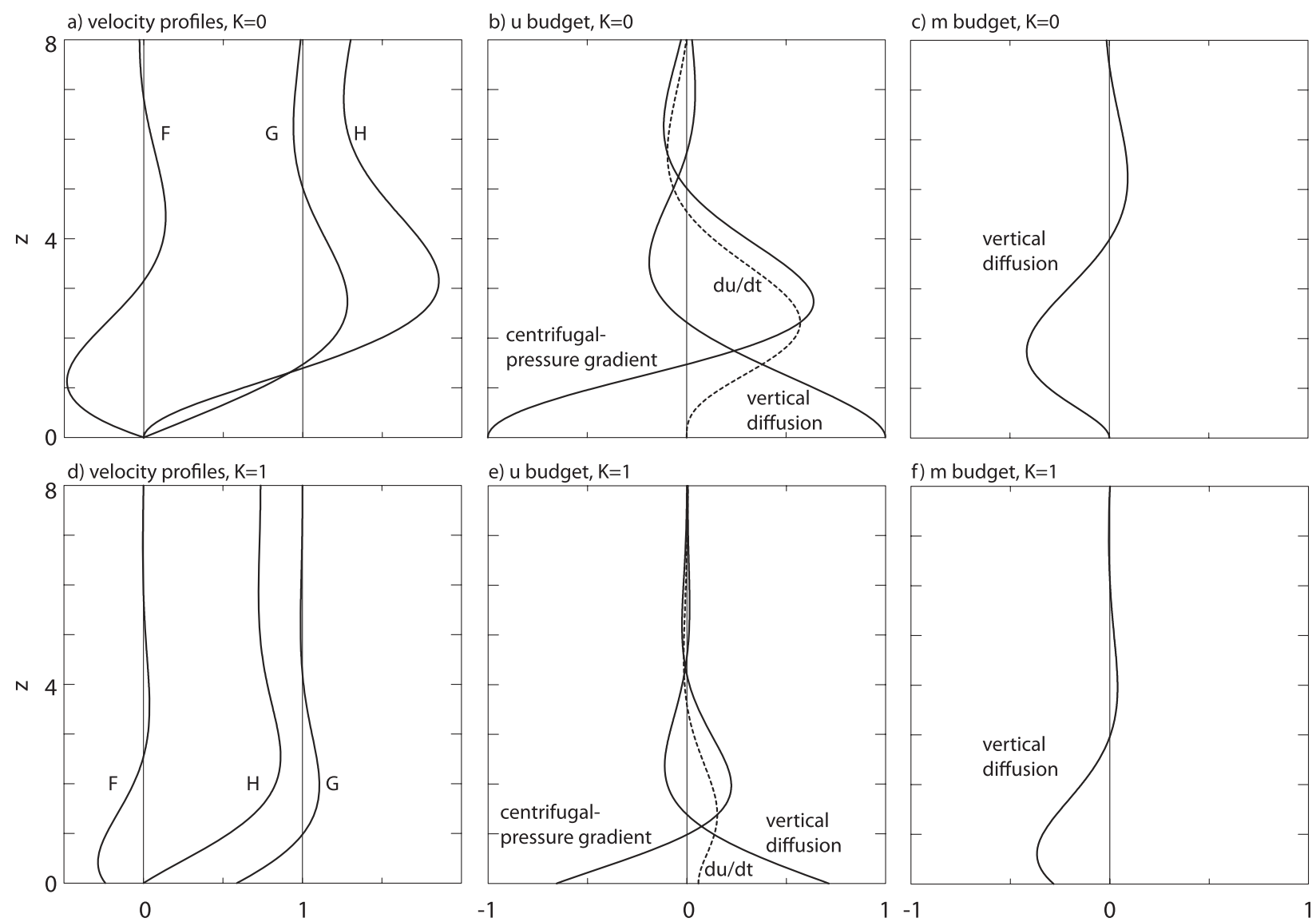

FIG. 5. Similarity solutions and their budget analyses for (a)-(c) the Bödewadt ( $K=0$, no-slip lower boundary condition) boundary layer and (d)-(f) the K71 ( $K=1$, partial-slip lower boundary condition) boundary layer.

The foregoing analysis suggests that traditional boundary layer theory can describe much about the vertical structure of the solutions in the inner core of the simulated hurricane. However, that analysis also reminds us that the success of boundary layer theory depends critically on the knowledge of an interior flow $V_{\infty}(r)$. In the
BR09b simulations, the strong variation of the presumed interior flow, $V_{g, m}$, with $l_{h}$ (Fig. 1d) suggests that horizontal diffusion, which is not included in traditional boundary layer theory, may act as a governor on $V_{\infty}(r)$ by reducing the angular momentum of parcels in the inner-core boundary layer and thereby smoothing the

TABLE 1. Major features of the K71 similarity solutions as a function of $K$ including the maximum azimuthal velocity $v_{m}$ and its height above the lower surface $z_{m}$, the maximum (negative) inflow velocity $u_{m}$ and its height $z_{m}^{u}$, the vertical velocity at large $z$, and the surface values of $v$ and $u$. The inflow angle $\beta=-\tan ^{-1} u(0) / v(0)$.

\begin{tabular}{|c|c|c|c|c|c|c|c|c|c|c|}
\hline$K$ & $v_{m}$ & $z_{m}$ & $K z_{m}$ & $u_{m}$ & $z_{m}^{u}$ & $w(\infty)$ & $v(0)$ & $u(0)$ & $\beta\left({ }^{\circ}\right)$ & $z_{m} / z_{m}^{u}$ \\
\hline 0.00 & 1.28 & 2.75 & 0.00 & -0.48 & 1.15 & 1.26 & 0 & 0 & - & 2.4 \\
\hline 0.25 & 1.24 & 2.45 & 0.61 & -0.45 & 0.90 & 1.20 & 0.19 & -0.17 & 42 & 2.7 \\
\hline 0.50 & 1.18 & 2.25 & 1.13 & -0.39 & 0.65 & 1.04 & 0.37 & -0.24 & 34 & 3.5 \\
\hline 0.75 & 1.14 & 2.08 & 1.56 & -0.33 & 0.50 & 0.87 & 0.49 & -0.25 & 27 & 4.2 \\
\hline 1.00 & 1.11 & 1.98 & 1.98 & -0.29 & 0.40 & 0.74 & 0.59 & -0.24 & 22 & 5.0 \\
\hline 1.25 & 1.09 & 1.90 & 2.38 & -0.25 & 0.35 & 0.63 & 0.65 & -0.22 & 19 & 5.4 \\
\hline 1.50 & 1.07 & 1.85 & 2.78 & -0.22 & 0.30 & 0.55 & 0.70 & -0.21 & 16 & 6.2 \\
\hline 1.75 & 1.06 & 1.81 & 3.17 & -0.20 & 0.25 & 0.48 & 0.74 & -0.19 & 14 & 7.2 \\
\hline 2.00 & 1.05 & 1.78 & 3.56 & -0.18 & 0.24 & 0.43 & 0.77 & -0.17 & 13 & 7.4 \\
\hline 2.25 & 1.05 & 1.76 & 3.96 & -0.17 & 0.21 & 0.39 & 0.79 & -0.16 & 12 & 8.4 \\
\hline 3.00 & 1.04 & 1.72 & 5.16 & -0.14 & 0.16 & 0.31 & 0.84 & -0.13 & 9 & 10.8 \\
\hline 4.00 & 1.03 & 1.68 & 6.72 & -0.11 & 0.12 & 0.23 & 0.88 & -0.11 & 7 & 14.0 \\
\hline
\end{tabular}



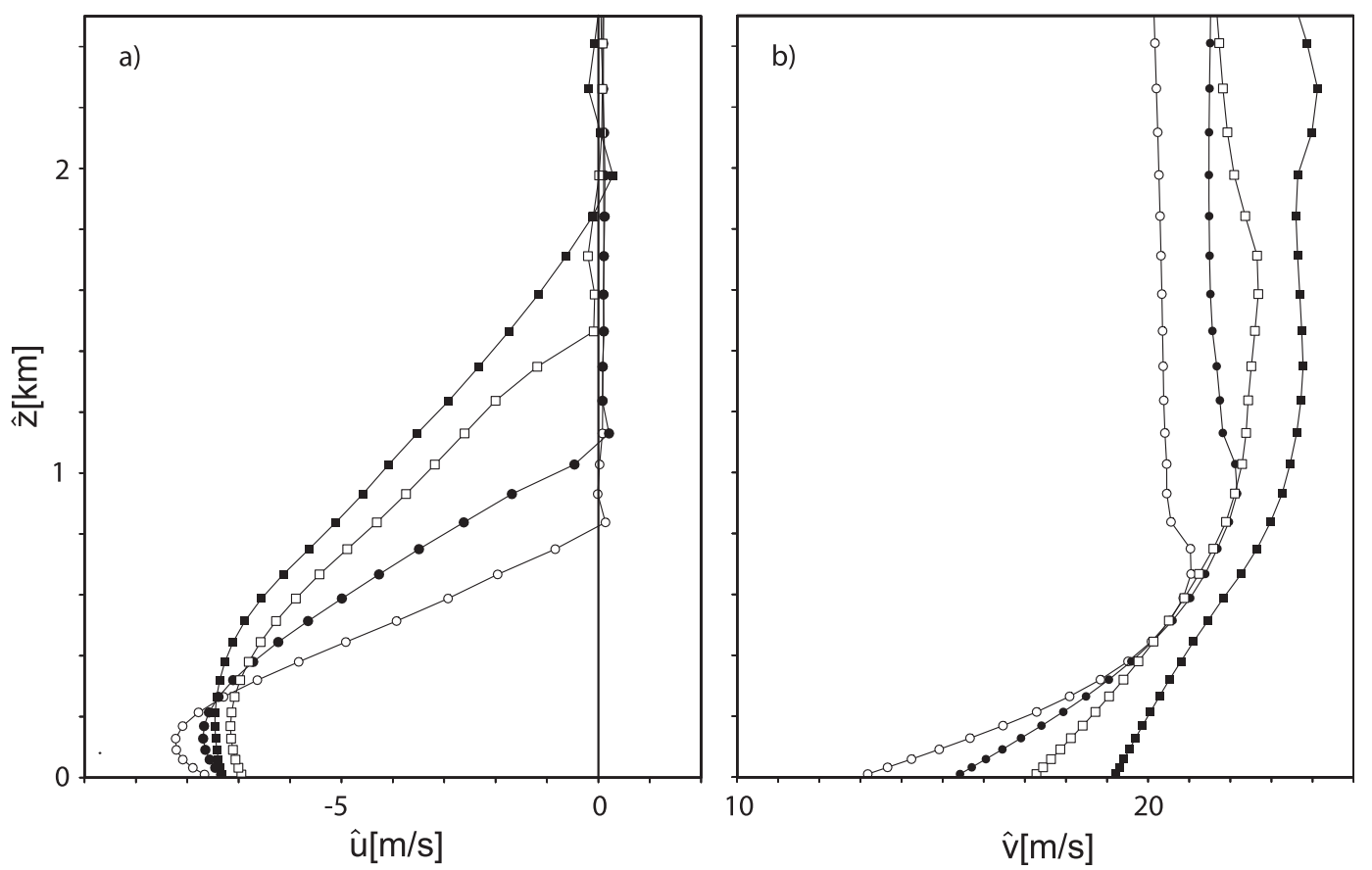

FIG. 6. Profiles at $\hat{r}=100 \mathrm{~km}$ of (a) $\hat{u}$ and (b) $\hat{v}$ for $l_{v}=25 \mathrm{~m}$ (open circles), $l_{v}=50 \mathrm{~m}$ (filled circles), $l_{v}=100 \mathrm{~m}$ (open squares) and $l_{v}=200 \mathrm{~m}$ (filled squares) for the case $l_{h}=1500 \mathrm{~m}$.

radial distribution of angular momentum carried upward (Figs. 3g,h); in this way horizontal diffusion may adjust $V_{\infty}(r)$ to a value consistent with that implied by the boundary layer solution. We believe this process is a good example of the interactive nature of rotating-flow boundary layers described in section 1 . Effects of horizontal diffusion will be further discussed in sections 5 and 6.

\section{b. Outer-core region $\left(\hat{r} \gg \hat{r}_{m}\right)$}

The outer-core region has much reduced interior flow velocities and so one might expect Ekman layer dynamics to apply there. As the Ekman layer is the boundary layer for a flow in solid-body rotation over a lower boundary having nearly the same rotation rate, it is essentially the linearized version of the Bödewadt boundary layer. Eliassen (1971) solves for the Ekman layer in cylindrical coordinates using a partial-slip lower boundary condition. Eliassen and Lystad (1977) study more general interior flows $V_{\infty}(r)$ through a heuristic analysis leading to their result for the vertical scale $\sqrt{\nu / I}$, where $I^{2}=r^{-3} \partial_{r}\left(r V_{\infty}\right)^{2}$ and $\nu$ is the eddy viscosity.

For typical parameter values, the partial-slip Ekman solutions of Eliassen and Lystad (1977, their Fig. 4) indicate $\hat{u}(\hat{z})$ increasing from a negative minimum near the surface to zero in the interior and $\hat{v}(\hat{z})$ increasing from its surface value to its interior value with very slight overshoot in both components. In a further elaboration on the Ekman-type model, Kepert (2001) solves for degree of overshoot [his (27) and Fig. 1] and finds it is in the range of $2 \%-4 \%$. The profiles for $\left[\hat{u}\left(\hat{z} ; l_{v}\right), \hat{v}\left(\hat{z} ; l_{v}\right)\right]$ for $l_{h}=1500 \mathrm{~m}$ for the BR09b simulations are shown in Fig. 6 for the outer-core region $(\hat{r}=100 \mathrm{~km})$ and are fairly well captured by the latter descriptions.

Kepert (2001) derives the height of the Ekman layer [his (27)]. Using the partial-slip, linearized-Bödewadt solution given by (4.10) of K71 we arrive at the same formula [ $K=\chi^{-1}$ in Kepert (2001)'s notation],

$$
z_{m}(K)=\pi-\tan ^{-1}(1+2 K)
$$

which gives $z_{m}(0)=3 \pi / 4=2.35$ and $z_{m}(4)=1.68$ and thus compares favorably with the values listed for the nonlinear K71 solution (Table 1). Replacing $\omega$ in (16) and (17) by an $I_{0}$ characterizing the outer-core region and applying the same logic as that following (17), we deduce that the linear Ekman solution also predicts $\hat{z}_{m}$ to increase with $l_{v}$ in the outer-core region, consistent with Fig. 6.

\section{Analysis of solution variation with $\boldsymbol{l}_{\boldsymbol{h}}$}

Figure 4 indicates that in the inner-core boundary layer, horizontal advection dominates vertical advection of $\hat{m}$ near the level of maximum horizontal diffusion $(\hat{z} \approx 0.5 \mathrm{~km})$. Here we consider the consequences of a 
model that balances horizontal advection with horizontal diffusion of angular momentum [i.e., a simplified form of (3) and (8)], which, with the definition of vorticity $\zeta=r^{-1} \partial_{r} m$, is

$$
\operatorname{Re}_{h} u(r) \zeta=\frac{\partial \zeta}{\partial r}
$$

where, in the same spirit as in the simple boundary layer solutions, we have assumed a constant viscosity $\left[f_{h}=\right.$ 1 in (8)].

Before examining solutions to (19) it will prove useful to first examine the BR09b numerical solutions just "upstream" of $\hat{z}_{m}$ which for cases with $l_{v}=200 \mathrm{~m}$ we take to be at $\hat{z}=0.5 \mathrm{~km}$; Figs. $7 \mathrm{a}-\mathrm{d}$ show respectively radial profiles of $\hat{u}, \hat{\zeta}, \hat{m}$, and $\hat{v}$ for $l_{h}=375,750,1500$, and $3000 \mathrm{~m}$. Beginning with Fig. 7a we observe a converging inflow that in all cases goes to zero at some finite radius $\hat{r}_{0}$; as $l_{h}$ becomes small it appears that $\hat{r}_{0}$ reaches an asymptotic limit of approximately $12 \mathrm{~km}$. Figure $7 \mathrm{~b}$ shows, consistent with (19), that $\hat{\zeta}$ takes its maximum value near $\hat{r}=\hat{r}_{0}$. Although $\hat{r}_{0}$ slightly increases with $l_{h}$, the main changes are the increasing width of the convergence zone, the diminishing peak strength of the vorticity and its outward spreading. Consistently, Figs. $7 \mathrm{c}$ and $7 \mathrm{~d}$ show the outward spreading of the distribution of $\hat{m}(r)$ and the diminishing peak intensity in $\hat{v}$ and its outward displacement.

There is a long history of solutions to (19) for profiles $u(r)$ that also satisfy (1), (2), and (5) with the constant viscosity forms of (6) and (7) (i.e., exact solutions to the Navier-Stokes equations; Craik 2009). In the present application, the flow is hydrostatic and, according to the analysis in Fig. 3c, horizontal diffusion plays a minor role in the radial momentum balance; hence we have some liberty in the choice for $u(r)$. For the radial velocity we consider the simplest function representing horizontal convergence and a stagnation point at $r_{0}$ :

$$
u(r)=\left\{\begin{array}{lll}
0 & \text { if } \quad r \leq r_{0} \\
-\frac{\gamma \lambda_{h}}{V}\left(r-r_{0}\right) & \text { if } \quad r \geq r_{0}
\end{array}\right.
$$

where $\gamma\left(\mathrm{s}^{-1}\right)$ is the specified constant rate of convergence. Although $\hat{\zeta} \rightarrow f$ (the Coriolis parameter $=0.5 \times$ $10^{-4} \mathrm{~s}^{-1}$ in the BR09b simulation) as $\hat{r} \rightarrow \infty$, Fig. $7 \mathrm{~b}$ suggests that letting $\hat{\zeta}(\infty) \rightarrow 0$ is a reasonable approximation, at least in the vicinity of the radius of maximum winds. With the latter condition and (20), (19) can be integrated once to obtain

$$
\zeta(r)=\zeta_{0} \exp \left[-\frac{\gamma \lambda_{h}^{2}}{2 \tilde{\nu}_{h}}\left(r-r_{0}\right)^{2}\right]
$$

where the definition of $\mathrm{Re}_{h}$ in (10) has been used and $\zeta_{0} \equiv \zeta\left(r_{0}\right)$, which is as yet unknown. Setting the argument of the exponential to -1 in (21), one can deduce the viscous length scale $\lambda_{h}=\sqrt{2 \tilde{\nu}_{h} / \gamma}$, which is typical of solutions of the type under consideration (e.g., Drazin and Riley 2006, p. 82). Adapting as before this constant viscosity solution to the variable viscosity BR09b numerical solutions, we expect that $\tilde{\nu}_{h} \sim l_{h}^{2} V / \lambda_{h}$ and that $\gamma \sim V / \lambda_{h}$, and that therefore $\lambda_{h} \sim l_{h}$, as expected from (12). As a simple check on this scaling we computed from Fig. $1 \mathrm{~b}$ the quantity $\delta \hat{r} \equiv \hat{r}_{m}\left(l_{h}, l_{v}\right)-\hat{r}_{m}\left(l_{h}=94 \mathrm{~m}\right.$, $\left.l_{v}\right)$ with the supposition that $\hat{r}_{m}\left(l_{h}=94 \mathrm{~m}, l_{v}\right)$ represents the inviscid limit; the result for $\ln (\delta \hat{r})$ plotted against $\ln \left(l_{h}\right)$, shown in Fig. 8, indicates an exponent of 1.1, which is close to the expected value of 1.0.

To complete the solution for $m(r)$ we need to use the inner boundary condition $m(0)=0$; here we assume that $\zeta(r)=\zeta_{0}$ for $0 \leq r \leq r_{0}$ (solid-body rotation) which, given (20), is a solution of (19). With the inner condition $m(0)=0$ and the outer condition $m(\infty)=m_{\infty}$ (a constant), it is clear that $m_{\infty}=\int_{0}^{\infty} r^{\prime} \zeta\left(r^{\prime}\right) d r^{\prime}=0.5 \zeta_{0}\left(r_{0}^{2}+r_{0} \sqrt{\pi}+1\right)$ and that therefore we know $\zeta_{0}$ in terms of $m_{\infty}$. The solution for $m(r)$ is therefore

$$
m(\xi)= \begin{cases}\frac{1}{2} \zeta_{0} r_{0}^{2} \xi^{2} & \text { if } \quad \xi \leq 1 \\ \frac{1}{2} \zeta_{0}\left\{r_{0}^{2}+r_{0} \sqrt{\pi} \operatorname{erf}\left[r_{0}(\xi-1)\right]+1-\exp \left[-r_{0}^{2}(\xi-1)^{2}\right]\right\} & \text { if } \quad \xi \geq 1\end{cases}
$$

where $\xi=r / r_{0}$. The solution for $m(r) / m_{\infty}$ [and the corresponding $v(r)]$ is shown in Fig. 9a for several values of $r_{0}$, which we recall is $\hat{r}_{0} / \sqrt{2 \tilde{\nu}_{h} / \gamma}$, the ratio of the stagnation radius to the viscous length scale. The qualitative similarity of these analytic solutions as a function of changing viscosity to the BR09b simulations (Figs. 7c,d) is apparent.
Up to this point we have not discussed thermodynamics. As mentioned in the introduction, the hurricane vortex boundary is distinguished from other rotatingflow boundary layer problems by the importance of baroclinicity in the interior flow. In preparation for the discussion in the next section of the present results as they relate to the E86 theory, we show in Figs. 7e and $7 \mathrm{f}$ 

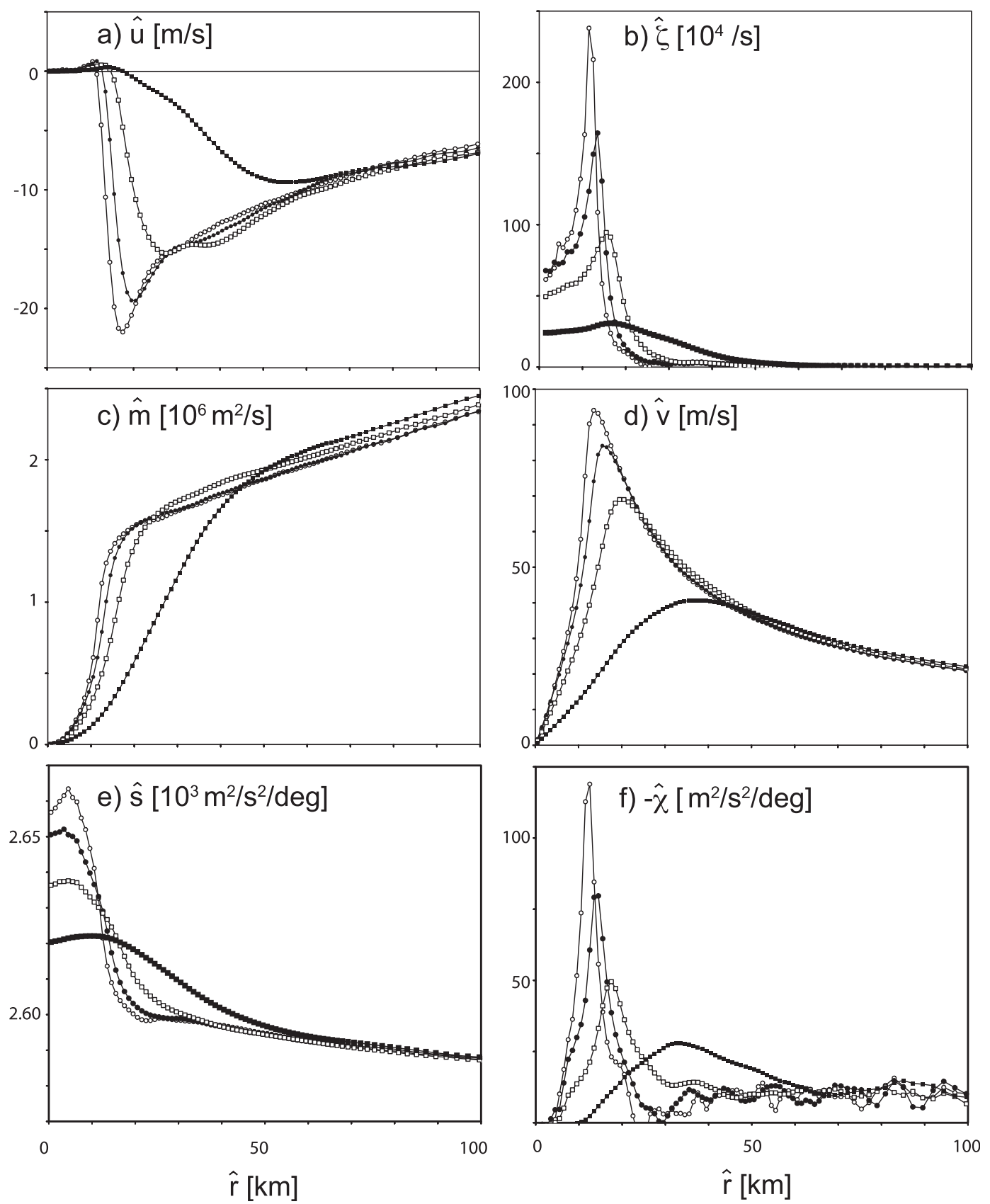

FIG. 7. Profiles at $\hat{z}=0.5 \mathrm{~km}$ of (a) $\hat{u}$, (b) $\hat{\zeta}$, (c) $\hat{m}$, (d) $\hat{v}$, (e) $\hat{s}$, and (f) $-\hat{\chi}$ for $l_{h}=375 \mathrm{~m}$ (open circles), $l_{h}=750 \mathrm{~m}$ (filled circles), $l_{h}=1500 \mathrm{~m}$ (open squares), and $l_{h}=1500 \mathrm{~m}$ (filled squares) for the case $l_{v}=200 \mathrm{~m}$.

the radial distribution of the entropy $\hat{s}$ and the quantity $\hat{\chi}=\hat{r} \partial_{\hat{r}} \hat{s}$ (which plays an analogous role to $\hat{\zeta}$ in the horizontal diffusion term) at $\hat{z}=0.5 \mathrm{~km}$ for the cases with $l_{v}=200 \mathrm{~m}$ and $l_{h}=375,750,1500$, and $3000 \mathrm{~m}$, respectively. As for $\hat{\zeta}$, the general correspondence between the location of the maximum in $-\hat{\chi}$ and $r_{0}$ is evidence that the horizontal advection-diffusion balance is roughly correct.
Following a similar route to the derivation of (22) by balancing horizontal advection and diffusion in (4) and using the radial velocity in (20), the variable $\chi(r)=r \partial_{r} b$ can be expressed in an analogous manner to $\zeta(r)$ in (21), namely $\chi=\chi_{0} \exp \left[-r_{0}^{2}(\xi-1)^{2}\right]$. To complete the solution for $b(\xi)$, we take the overall change in $b, \Delta b$, as a given, so that $\chi_{0}=\Delta b\left\{\int_{1}^{\infty} \xi^{\prime-1} \exp \left[-r_{0}^{2}\left(\xi^{\prime}-1\right)^{2}\right] d \xi^{\prime}\right\}^{-1}$. The solution analogous to (22) is thus 


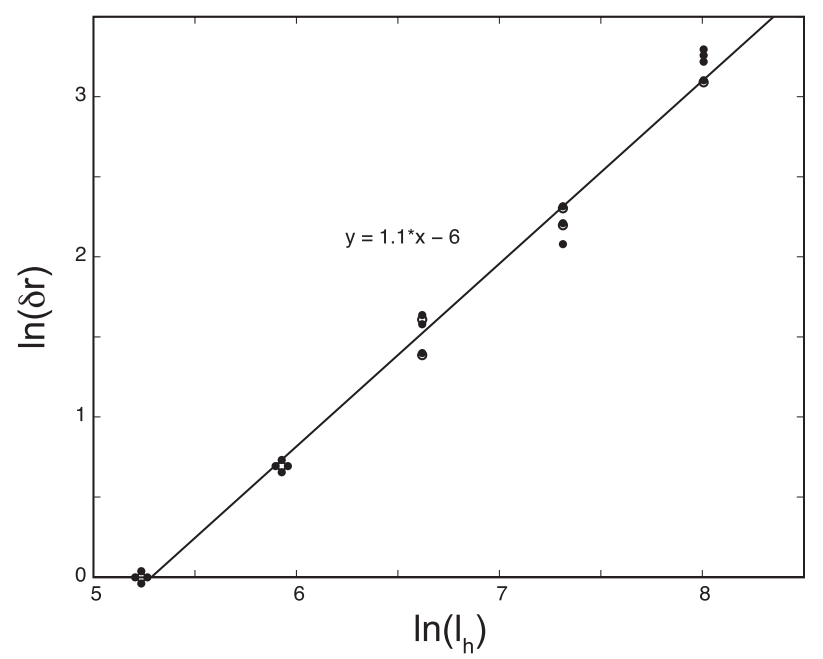

FIG. 8. Analysis of the dependence of the distance $\delta \hat{r} \equiv\left[\hat{r}_{m}\left(l_{h}\right)-\right.$ $\left.\hat{r}_{m}\left(l_{h}=94 \mathrm{~m}\right)\right]$ on $l_{h}$ for the cases $l_{h}=188,375,750,1500$, and $3000 \mathrm{~m}$ and $l_{v}=25,50,100,200$, and $400 \mathrm{~m}$. Points that are superimposed are slightly displaced from the actual position, indicated by the open circles.

$$
b(\xi)=\left\{\begin{array}{lll}
\Delta b & \text { if } & \xi \leq 1 \\
\chi_{0} \int_{\xi}^{\infty} \xi^{\prime-1} \exp \left[-r_{0}^{2}\left(\xi^{\prime}-1\right)^{2}\right] d \xi^{\prime} & \text { if } & \xi \geq 1
\end{array}\right.
$$

and is plotted in Fig. $9 \mathrm{~b}$ with $\Delta b=v_{m}^{2}{ }^{6}$ The qualitative correspondence between this analytical solution and the BR09b simulations (Fig. 7e) is evident.

\section{Horizontal diffusion and the E86 theory}

The E86 theory has been critically reviewed in Smith et al. (2008, hereafter SMV) and in BR09a. The basic elements of the E86 theory are gradient wind/hydrostatic balance and conservation of $\hat{m}$ and $\hat{s}$ above the boundary layer and a mixed layer model to obtain a relation of the form $(d \hat{s} / d \hat{m})_{z=h}=g(\hat{s}, \hat{m})$, where $h$ is the top of the boundary layer. According to SMV, "a major deficiency of the E86 theory is the tacit assumption of gradient wind balance in the boundary layer." BR09a find that, in the limit of small horizontal diffusion, numerical solutions exhibit gradient wind imbalance in and above the boundary layer, while for larger values of horizontal diffusion, they find that nongradient wind effects are minimal (see Fig. 12 of BR09a).

Based on the present analysis we offer the following interpretation. The E86 theory and its later extensions

\footnotetext{
${ }^{6}$ Based on a volume integration of the relevant thermal-wind equation $\left(\partial_{r} b=r^{-1} \partial_{z} v^{2}\right)$, it may be shown that $\Delta b \propto v_{m}^{2}$.
}
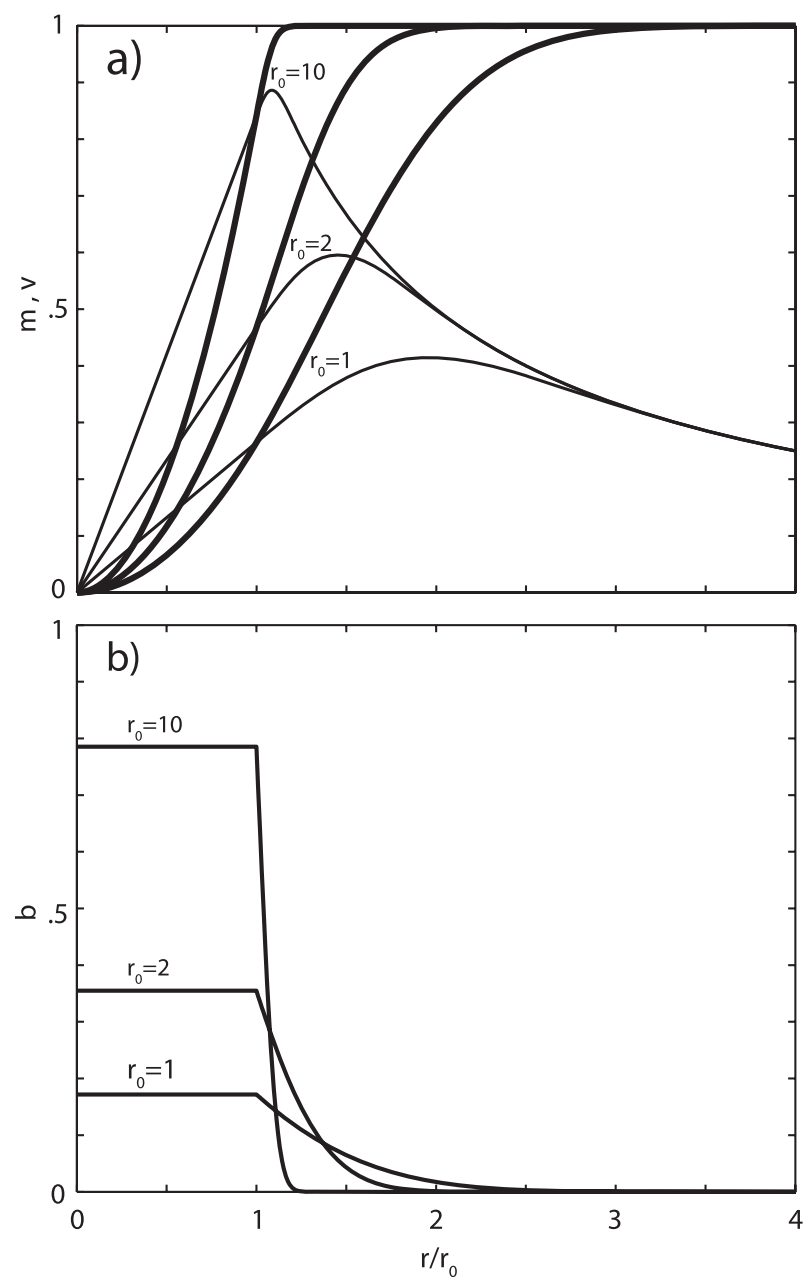

FIG. 9. Analytical solution for (a) $m(r)$ (thick lines) and $v(r)$ (thin lines) and (b) $b$ for $r_{0}=1,2$, and 10; larger $r_{0}$ solutions correspond to cases with smaller viscosity.

(most recently Emanuel and Rotunno 2011) is a theory for the "interior" flow. The theory uses a mixed layer model, with no assumption of gradient wind balance, to derive $(d \hat{s} / d \hat{m})_{z=h}=g(\hat{s}, \hat{m})$. Up to this point the E86 theory is both physically and mathematically selfconsistent. To take the theory a step further, SMV are correct in their criticism that the radial velocity should not be obtained by a simple inversion of the $\hat{m}$ mixed layer equation, but rather a system of boundary layer equations must be solved. Based on the evidence presented herein, that system would be the steady version of (1)-(5) with $\alpha=1 / \sqrt{\mathrm{Re}_{v}} \ll 1$ and $\mathrm{Re}_{h}=1$; the interior flow would appear explicitly through the radial pressure gradient in (1) and through the upper boundary conditions on $(\hat{m}, \hat{s})$.

A boundary layer calculation of the type suggested would in general need to be followed by a recalculation of the interior flow to accommodate the distributions of 
$(\hat{m}, \hat{s})$ transported upward from the boundary layer; the recalculated interior flow would in turn require a recalculation of the boundary layer and so on until boundary layer and interior flow are mutually adjusted. This process of mutual adjustment for barotropic rotatingflow boundary layers is well known in the fluid dynamics literature (Rott and Lewellen 1966, 132-133). The major difference between the classic barotropic case and the baroclinic hurricane is that, in the former, the first approximation to the interior flow is usually given whereas in the moist, baroclinic hurricane, it must be constructed through considerable labor.

Based on the BR09b simulations with $l_{h} \geq 1000 \mathrm{~m}$, we conjecture that a boundary layer calculation including both partial-slip and horizontal diffusion would a) counter the tendency of the boundary layer $\hat{v}$ to overshoot $V_{\infty}$ and b) produce a smaller $V_{\infty}$ after a recalculation of the interior flow as the horizontally diffuse distribution of $\hat{m}$ is transported upward (Fig. 3h). Evidence for the latter point can be seen in Fig. 1f where there is a clear dependence of E-PI on (primarily) $l_{h}$ indicating an adjustment of both interior and boundary layer flow parameters.

\section{Comparison with observations}

Inasmuch as the parameters $\left(l_{v}, l_{h}\right)$ are not known from independent measurements, we look to some recent observations to see if reasonable choices $\left(l_{v}, l_{h}\right)$ can describe the data. Zhang et al. (2011) analyzed a great number of dropsondes to produce a composite picture of the radial and azimuthal boundary layer hurricane velocities. Our Fig. 10 shows several cases from the BR09b simulations in the same manner as was done in Fig. 10 of Zhang et al. (2011) with the radius nondimensionalized by $\hat{r}_{m}$, the radial velocity by the absolute value of its minimum, and the azimuthal velocity by its maximum; the height scale is dimensional. Cases with $l_{v}=50-100 \mathrm{~m}$ seem to produce very comparable vertical structures to those observed but for brevity we show here just cases with $l_{v}=50 \mathrm{~m}$. The cases with $l_{h}=750$ and $1500 \mathrm{~m}$ and $l_{h}=300 \mathrm{~m}$ have almost identical structure when plotted in this manner and provides another piece of evidence that the scaling (12) is correct; the primary difference between these cases is in the scaling velocities $\hat{u}_{\min }$ and $\hat{v}_{\max }$.

At a more detailed level, Fig. 10 indicates that the ratio of the heights of maximum azimuthal and radial velocities $\hat{z}_{m} / \hat{z}_{m}^{u}$ is roughly 7 , which is in agreement with the Zhang et al. (2011) composites. Furthermore, Fig. 10 indicates that the surface inflow angle $\beta$ at $\hat{r}_{m}$ is $28^{\circ}, 22^{\circ}$, and $22^{\circ}$ for the cases when $l_{h}=750,1500$, and $3000 \mathrm{~m}$, respectively, while the Zhang et al. (2011) composite for all storms has $\beta \approx 24^{\circ}$, which is in agreement with Powell et al. (2009) in which an average inflow angle $\beta \approx 23^{\circ}$ was found from dropsonde data. We note from Table 1 that the $\mathrm{K} 71$ solutions have $z_{m} / z_{m}^{u} \approx 7$ for $K=1.75$, for which $v_{m}=1.06$, and that $\beta \approx 23^{\circ}$ for $K=1$, for which $v_{m}=1.11$; these data add further support to the idea that overshoot in natural storms is probably modest.

While the BR09b experiments were conducted following earlier studies with the ratio of enthalpy to momentum flux coefficients $C_{k} / C_{d}=1.0$, recent studies indicate a ratio that depends on wind speed and asymptotic values closer to 0.5 for large wind speeds (Haus et al. 2010; Bell 2010). Bryan (2012) has conducted a parameter sensitivity experiment varying $l_{h}, l_{v}$ and $C_{k} / C_{d}$ over wide ranges and finds that the settings $l_{h} \approx$ $1000 \mathrm{~m}, l_{v} \approx 50 \mathrm{~m}$, and $C_{k} / C_{d} \approx 0.5$ produce the most reasonable match by a variety of measures of the present axisymmetric model solutions to observational studies of hurricanes.

Although the boundary layer parameterization used by BR09b is much simpler than the more complex schemes that have been used (Braun and Tao 2000; Smith and Thomsen 2010; Nolan et al. 2009a,b), the comparisons made here suggest that solutions accurate to within observational uncertainty are produced for certain combinations of the mixing lengths $\left(l_{v}, l_{h}\right)$.

\section{Conclusions}

In the present paper we have taken a closer look at the effects of parameterized diffusion on the nearly steady axisymmetric numerical simulations of hurricanes presented in BR09a. In that paper it was concluded that horizontal diffusion was the most important control factor on the maximum simulated hurricane intensity. Through budget analysis (section 3) we have shown further that horizontal diffusion is a major contributor to the angular momentum budget, primarily in the hurricane boundary layer. We have provided a new scale analysis (section 2), one that explicitly recognizes the anisotropic nature of the parameterized model diffusion, showing why the horizontal diffusion plays such a dominant role. A simple analytical model (section 5), we believe, captures the essence of the effect.

A detailed examination of the role of vertical diffusion in the BR09b simulations (section 4) shows that the boundary layer in these simulations is consistent with known analytical solutions. Specifically for fixed $l_{h}$, the BR09b boundary layers increase in depth and decrease in the amount of "overshoot" $\hat{v}_{m} / \hat{v}_{g, m}$ with increasing $l_{v}$. However, $\hat{v}_{m}$ itself is relatively insensitive to $l_{v}$ and the overshoot variation with $l_{v}$ mainly comes from changes in $\hat{v}_{g, m}$ with $l_{v}$. Both $\hat{v}_{m}$ and $\hat{v}_{g, m}$ are strong decreasing functions of $l_{h}$. 


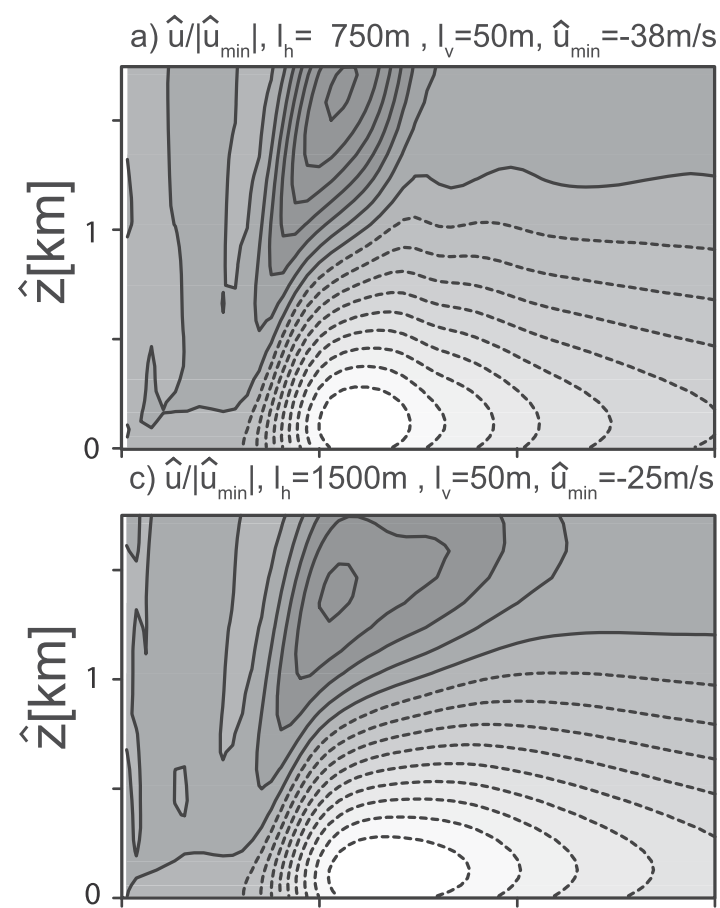

b) $\hat{v} / \hat{v}_{m}, I_{h}=750 m, I_{v}=50 m, \hat{v}_{m}=86 \mathrm{~m} / \mathrm{s}$

e) $\hat{u} /\left|\hat{u}_{\min }\right|, I_{h}=3000 \mathrm{~m}, \mathrm{I}_{\mathrm{v}}=50 \mathrm{~m}, \hat{u}_{\min }=-16 \mathrm{~m} / \mathrm{s}$

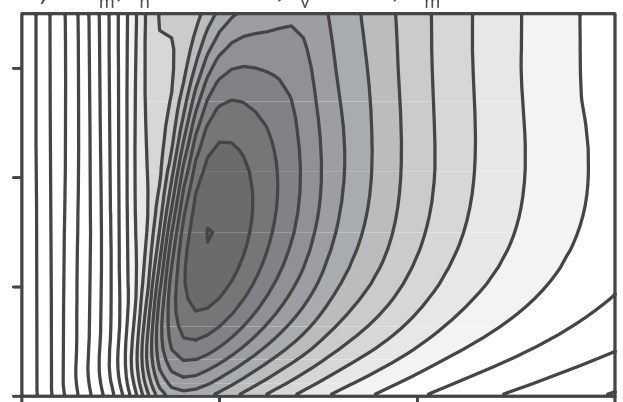

d) $\hat{v} / \hat{v}_{m}, I_{h}=1500 m, I_{v}=50 m, \hat{v}_{m}=66 \mathrm{~m} / \mathrm{s}$

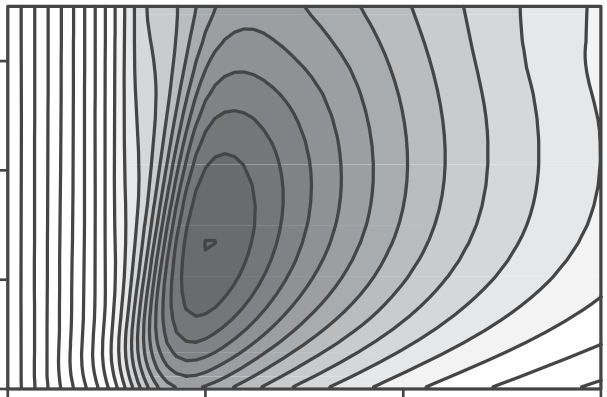

f) $\hat{v} / \hat{v}_{m}, I_{h}=3000 m, I_{v}=50 m, \hat{v}_{m}=43 \mathrm{~m} / \mathrm{s}$
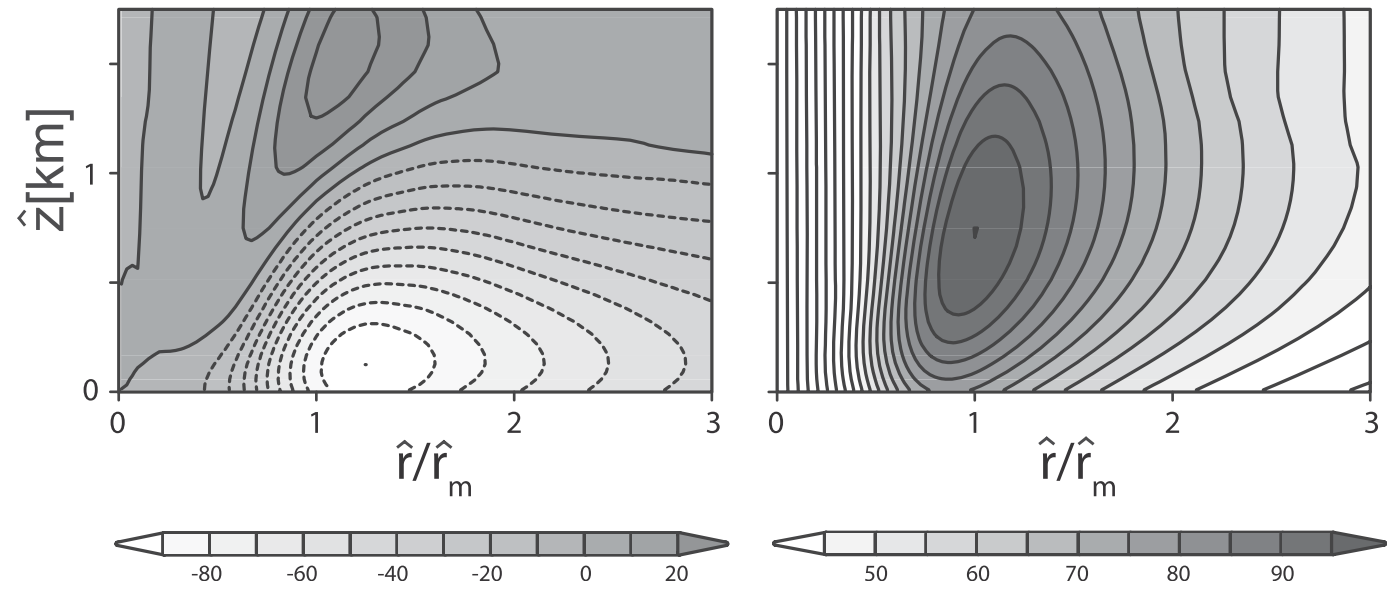

FIG. 10. Solutions for radial and azimuthal velocity normalized as in the composite observations shown in Fig. 10 of Zhang et al. (2011) for (a),(b) $l_{h}=750 \mathrm{~m}, l_{v}=50 \mathrm{~m}$, (c),(d) $l_{h}=1500 \mathrm{~m}, l_{v}=50 \mathrm{~m}$, and (e),(f) $l_{h}=3000 \mathrm{~m}, l_{v}=50 \mathrm{~m}$. Contour interval $=0.1$ for the radial velocity and 0.05 for the azimuthal velocity. Color bar labeled in percentage.

Finally we offer an interpretation of the discussion in the literature of the E86 theory and its relation to boundary layer dynamics (section 6). We argue that the E86 theory (and its extensions) is for the interior flow $V_{\infty}(r, z)$ (i.e., the flow away from the lower frictional boundary); the theory relies on a mixed-layer model to obtain a relation between entropy and angular momentum at the boundary layer top but should be considered as being silent on the radial-vertical boundary layer flow. In agreement with SMV we believe to obtain the radial-vertical boundary layer flow consistent with the E86 outer flow, a boundary layer model should used; however the evidence presented here suggests that horizontal diffusion be included in that boundary layer model. If horizontal diffusion effects are strong enough to counteract the tendency for overshooting, the E86 model plus the boundary layer correction give a reasonably accurate picture of the nearly steady axisymmetric hurricane. Observational evidence (Emanuel 2000; Bell and Montgomery 2008; see also section 7 herein) suggests that the latter view is not far from the truth. 
Although the nearly steady axisymmetric numerical model of a hurricane is a considerable simplification of a very complex three-dimensional, time-dependent reality, it is still complex enough to provoke a continuing discussion in the literature of its basic mechanics. In this spirit the present paper has analyzed the far-ranging effects of diffusion in the simulated hurricane boundary layer. In a recent study Emanuel and Rotunno (2011) investigated the possible effects on steady-state vortex structure of turbulent mixing in the hurricane upperoutflow layer looking at how adjustments at remote distances can affect the low-level vortex structure. Clearly the steady-state axisymmetric model has yet to give up all its secrets.

Acknowledgments. The authors thank Dr. C. Davis (NCAR) for his comments on the first draft of this manuscript. The critical comments of reviewer David Nolan (University of Miami) and another anonymous reviewer are gratefully acknowledged. This work was sponsored in part by the Office of Naval Research, Prime Contract N00014-10-1-0148 awarded to York University, as part of the National Oceanographic Partnership Program.

\section{APPENDIX}

\section{Similarity Solutions Following K71}

The boundary layer for a fluid of infinite radius in solidbody rotation possesses a similarity solution (Schlichting 1968, 213-218). The radial, azimuthal, and vertical equations of motion become (11.6) of Schlichting (1968) for the similarity functions $F(z), G(z), H(z)$ representing the radial, azimuthal, and vertical velocities, respectively. To allow for partial slip following K71, the lower boundary condition in (11.7) of Schlichting (1968) is replaced by (14), which translates to

$$
z=0: F=K F^{\prime}, \quad G=K G^{\prime}, \quad H=0,
$$

where the prime denotes differentiation with respect to the similarity coordinate $z$.

The numerical solution for $(F, G, H)$ is found following the procedure outlined in White (1974, 163-170) for the analogous problem of the boundary layer on disc of infinite radius rotating below a flow with no horizontal motion as $z \rightarrow \infty$. The numerical procedure is basically a "shooting" method in which one guesses the derivatives of $F$ and $G$ at $z=0$ and then integrates (here using the Runge-Kutta method) the nonlinear ordinary differential equations to a large value of $z=z_{l}$ and tries to "hit" the boundary conditions as $z \rightarrow \infty$; the guess is corrected using the Newton-Raphson method and the procedure continued until convergence is reached. For the calculations reported herein $z_{l}=10$ and double precision was required for satisfactory convergence of the solutions.

\section{REFERENCES}

Batchelor, G. K., 1967: An Introduction to Fluid Dynamics. Cambridge University Press, $615 \mathrm{pp}$.

Bell, M. M., 2010: Air-sea enthalpy and momentum exchange at major hurricane wind speeds. Ph.D. dissertation, Naval Postgraduate School, 133 pp.

— and potential intensity of category 5 Hurricane Isabel (2003) from 12 to 14 September. Mon. Wea. Rev., 136, 2023-2046.

Braun, S. A., and W.-K. Tao, 2000: Sensitivity of high-resolution simulations of Hurricane Bob (1991) to planetary boundary layer parameterizations. Mon. Wea. Rev., 128, 3941-3961.

Bryan, G. H., 2012: Effects of surface exchange coefficients and turbulence length scales on the intensity and structure of numerically simulated hurricanes. Mon. Wea. Rev., 140, 1125-1143.

_ the maximum intensity of tropical cyclones. J. Atmos. Sci., 66, 3042-3060.

$\longrightarrow$, and - 2009b: The maximum intensity of tropical cyclones in axisymmetric numerical model simulations. Mon. Wea. Rev., 137, 1770-1789.

Craik, A. D. D., 2009: Exact vortex solutions of the Navier-Stokes equations with axisymmetric strain and suction or injection. J. Fluid Mech., 626, 291-306.

Drazin, P. G., and N. Riley, 2006: The Navier-Stokes Equations: A Classification of Flows and Exact Solutions. Cambridge University Press, $196 \mathrm{pp}$.

Eliassen, A., 1971: On the Ekman layer in a circular vortex. J. Meteor. Soc. Japan, 49, 784-789.

_ , and M. Lystad, 1977: The Ekman layer of a circular vortex-A numerical and theoretical study. Geophys. Norv., 31,1-16.

Emanuel, K. A., 1986: An air-sea interaction theory for tropical cyclones. Part I: Steady-state maintenance. J. Atmos. Sci., 43, 585-604.

_ 1997: Some aspects of hurricane inner-core dynamics and energetics. J. Atmos. Sci., 54, 1014-1026.

_ 2000: A statistical analysis of tropical cyclone intensity. Mon. Wea. Rev., 128, 1139-1152.

_ 2004: Tropical cyclone energetics and structure. Atmospheric Turbulence and Mesoscale Meteorology, E. Fedorovich, R. Rotunno, and B. Stevens, Eds., Cambridge University Press, 165-191.

_ - and R. Rotunno, 2011: Self-stratification of tropical cyclone outflow. Part I: Implications for storm structure. J. Atmos. Sci., 68, 2236-2249.

Haus, B. K., D. Jeong, M. A. Donelan, J. A. Zhang, and I. Savelyev, 2010: Relative rates of sea-air heat transfer and frictional drag in very high winds. Geophys. Res. Lett., 37, L07802, doi:10.1029/ 2009GL042206.

Kepert, J. D., 2001: The dynamics of boundary layer jets within the tropical cyclone core. Part I: Linear theory. J. Atmos. Sci., 58, 2429-2483.

Kuo, H. L., 1971: Axisymmetric flows in the boundary layer of a maintained vortex. J. Atmos. Sci., 28, 20-41.

Marks, F. D., P. G. Black, M. T. Montgomery, and R. W. Burpee, 2008: Structure of the eye and eyewall of Hurricane Hugo (1989). Mon. Wea. Rev., 136, 1237-1259. 
Nolan, D. S., J. A. Zhang, and D. P. Stern, 2009a: Evaluation of planetary boundary layer parameterizations in tropical cyclones by comparison of in situ observations and highresolution simulations of Hurricane Isabel (2003). Part I: Initialization, maximum winds, and the outer-core boundary layer. Mon. Wea. Rev., 137, 3651-3674.

, D. P. Stern, and J. A. Zhang, 2009b: Evaluation of planetary boundary layer parameterizations in tropical cyclones by comparison of in situ observations and high-resolution simulations of Hurricane Isabel (2003). Part II: Inner-core boundary layer and eyewall structure. Mon. Wea. Rev., 137, 3675-3698.

Persing, J., and M. T. Montgomery, 2003: Hurricane superintensity. J. Atmos. Sci., 60, 2349-2371.

Powell, M. D., E. W. Uhlhorn, and J. W. Kepert, 2009: Estimating maximum surface winds from hurricane reconnaissance measurements. Wea. Forecasting, 24, 868-883.

Rott, N., and W. S. Lewellen, 1966: Boundary layers and their interactions in rotating flows. Progress in Aeronautical Sciences, Pergamon Press, 111-144.

Rotunno, R., Y. Chen, W. Wang, C. Davis, J. Dudhia, and G. J. Holland, 2009: Large-eddy simulation of an idealized tropical cyclone. Bull. Amer. Meteor. Soc., 90, 1783-1788.
Schlichting, H., 1968: Boundary-Layer Theory. 6th ed. McGrawHill, 748 pp.

Skamarock, W. C., J. B. Klemp, J. Dudhia, D. O. Gill, D. M. Barker, W. Wang, and J. G. Powers, 2005: A description of the Advanced Research WRF version 2. NCAR Tech. Note NCAR/TN-468+STR, 88 pp.

Smith, R. K., and G. L. Thomsen, 2010: Dependence of tropicalcyclone intensification on the boundary layer representation in a numerical model. Quart. J. Roy. Meteor. Soc., 136, 1671-1685.

$\longrightarrow$, M. T. Montgomery, and S. Vogl, 2008: A critique of Emanuel's hurricane model and potential intensity theory. Quart. J. Roy. Meteor. Soc., 134, 551-561.

Taylor, G. I., 1915: Eddy motion in the atmosphere. Philos. Trans. Roy. Soc. London, A215, 1-26.

Vogl, S., and R. K. Smith, 2009: Limitations of a linear model for the hurricane boundary layer. Quart. J. Roy. Meteor. Soc., 135, 839-850.

White, F. M., 1974: Viscous Fluid Flow. McGraw-Hill, 725 pp.

Zandbergen, P. J., and D. Dijkstra, 1987: Von Kármán swirling flows. Annu. Rev. Fluid Mech., 19, 465-491.

Zhang, J. A., R. F. Rogers, D. S. Nolan, and F. D. Marks Jr., 2011: On the characteristic height scales of the hurricane boundary layer. Mon. Wea. Rev., 139, 2523-2535. 